

$T \mathbb{R} \mathbb{I} \mathbb{E}$

SOMMAIRE

\section{DES COQUILLES,}

TANT FLUVIATILES

QUE TERRESTRES;

QUI SE TROUVENT

\section{AUX ENVIRONS DE PARIS.}

PA M. GEOFFROY, Docteur, liégent de lá Faculcé de Médecine.

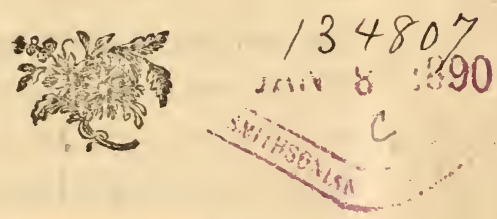

A PARIS;

Chez J. B. GUII. MUSIER fils, Libraire;

Quai des Auguftins, à S. Etienne.

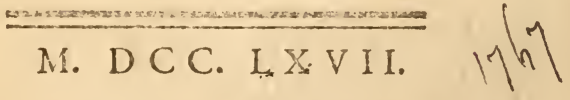

Aver Approbaion, E Privilége dir Roi. 


$$
\text { . }
$$


1763

AVIS DE L'AUTEUR.

A

Ès av oir publié il y a quelques années l'Hiftoire des Infectes des en. virons de Paris, mon projet étoit de continuer le même travail fur les Vers, \& de donner au Public l'Hiftoire de ces Animaux. La claffe des Vers fe rapproche de celle des Infectes, $\&$ devient d'autant plus intérefrante qu'elle eft peut-être la moins connue jufqu'ici. J'avois déja recueilli à ce fujet plufieurs obfervations qui paroiffoient curieures; j'efpérois les augmenter, rectifier cellesquiétoient défectueufes, réitérer l'examen de quel. ques autres que je ne regardois pas commesùres \& bien démontrées, \& donner, finon un Corps complet, au moins un Efai de lHifoire des Vers. Maisplus jaifait de recherches, plus

a ij 
les difficultés fe font accrues. Chaque genre de Vers, \& j'ofe prefque dire, chaque efpece, offre un objet tout à fait neuf, qui demande à lui feul.prefque autant de travail que leș claffes entieres des grands Animaux. A peine connoit on la plupart des Vers. Ceux mêmes que nous portons \& qui vivent dans le corps de l'Homme ne font pas encore parfaitement cunnus des $\mathrm{Na}$ turaliftes. Le tefte di Tænia \& fa configuration forment un problême en fait d'Hiftoire Naturelle; \& malgré les belles \& intéreffantes déconvertes de lilluftre M. Tremblay, on ne connoît prefque point les Polypes, ces efpeces de Vers fi finguliers, \& qui tiennent fi peu de la nature des Animaux. L'on eft incertain fi chaque $\mathrm{P}_{\mathrm{O}}$ lype eft un feul animal, ou un fimple fourreau qui renferme une famille 
entiere de Polypes. Quoique ces difficultés fuffent bien capables de m'arrêter, j'aurois cependant tâché de remplir au moins en partie mon deffein, fi cles occupations plus férieufes \&r plus intéreffantes ne m'en enfent détourné. Dans l'impoffibilité de fu:-vre ce travail, j'aicru devoirlaiffer à des Naturalittes qui auroient plus de loifre, le foin de remplir un projet qui me devient impraticable, \& qui eft une partie des plus difficiles de l'Hirtoire des Animaux. Je me fuis conten. té de mettre en ordre ce que j'avois. obfervé au fujet des Coquillages. Cette famille la plus nombreufe de la claffoules Ver's, n'eft pas la moins, intéreffante. Elle offre beaucoup de fingularités qui ne fe voyent point dans les autres claffes d'Animaux. D'ailleurs elle n'étoit point rangée:

aiij; 


\section{vj AVIS DE L'AUTEUR.}

fous un ordre affez méthodique pour en faciliter la connoiffance. J'ai donc penfé pouvoir hafarder de publier ce petit Traité des Coquillages qui fe trouvent aux environs de Paris. Si par la fuite quelque Naturalifte veut augmenter ce commencement d'obfervations, au fujet des Coquilles, $\& x$ y joindre l'Hiftoire des autres Animaux que renferme la clatse des Vers, ce fera un fervice important quil rendra aux Amateurs de lHifo toire Naturelle. 


\section{E X P L I C A T I O N.}

Des Noms abrégés des Auteurs cités. dans ce Traité.

Act, UPS 1736. Linnxi Animalia Suecix. in Actibus Upfalienfibus anni $1736: \operatorname{in}^{\circ} 4^{\circ}$. Adanfon, Seneg. Hiftoire Naturelle du Sénégal, par M. Adanfon. Paris; 17.57. in $4^{\circ}$. $\mathrm{fg}_{\mathrm{g}}$.

Aldrov. Exfang. Uly fis Aldrovandi libri IV. de Exfanguibus. Bonon. 1642. in fol. fig. Argenville Conchyl. L'Hiftoire Naturelle éclaircie dans une de fes parties; la Conchyliologie, premiere partie; la Zoomonphofe, feconde partie, par d'Argenville. Paris, 1757 . in-fol.fg.

Bonan. Recreat. Bonani Recreatio mentis 86. oculi, Roma, 1684. in-4. ${ }^{\circ}$.

Column Putpur. Fabii Columne Lyncai Opure culum de Purpura. Kilice, 1675. in- $4^{\circ}$, figa Dale Pharmac. Samuelis Dalei Pharmaco. logia. Lugd. Bat. 1739 .

Frifch. Inf. Joanh Leonard Frifch. Befchreia 
riij Explication des Noms abrégés

beng von Infeeten in Teutfchland. Berlin, i 720 . in $4^{\circ}$. fig.

Gefner, Aquat. Conradi Gefneri Hiftoria Animalium, de Pifcibus \& Aquatilibus. Francofurt. 1620. in fol.

It. Eland. Itinerarium $\mathbb{E}$ landicum, ou Voyage de Scanie, par M. Linnxus. Stockholm, i7so.

Klein Oftr. Jacobi Theodori Klein tentamen Methodi Oftracologicx; five Difpofitio naturalis Cochlidum \& Concharum in claites genera \& fpecies. Lugd. Bat. I7) 3. in $-4^{\circ}$. fig.

Linn. Faun. Suec. Caroli Linnxi Fauna Suecica Stocholmia, $774^{6}$. in- $^{\circ}$. fig.

Linn. Syft. Nat. edit. 10. C. Linnxi Syftema Natura', editio decirra reformata. $\mathrm{Hol}$. mia, $1759 . \mathrm{in} \cdot 8^{\circ} .2$ vol.

Lift. Angl. Martini Lifter Hiftoria Animalium Anglix. Londini, 1678. in. $4^{\circ}$. fig. Lift. Hift. M. Lifter Hiftoria Conchyliorum. Londini, 168 ;. in fol.

-Lift. Exerc. A nat. M. Lifter Exercitatio Anatomica de Cuchleis. Londini, 1694 . in- $8^{\circ}$. Alerret Pin. Chrift. Merret Pinax rerum 


\section{des Auceurs. ive}

Naturalium Britannicatum. Londini, 1667. in $-8^{\circ}$.

Petiv. Muf. Jacobi Petiveri, Centurix Múfri Petiveriani. Londini, 1695. in- $8^{\circ}$.

Swammerd. Babl. Nat. Joannis Swammerdam Biblia Naturx. Lugd. Bat. 1738. in fol.

Tulp. Obferv. Nicolai Tulpii Obfervationes Medicx. Amftel, 1641. in $8^{\circ}$ 
Membre de l'Académie Roiale des Sciences de Paris, de la Société Roiale de Londres. Cenfeur Roial.

I

"Ar lu par ordre de Monfeigneur le ViceChancelier, un manufcrit intitulé : Traité formaire des Coquilles des environs de Paris, \& je n'i ai rien trouvé qui puiffe en empêcher l'impreflion. L'Auteur paroît i remplir fon objet, qui eft d'infpirer aux jeunes Gens du gout pour l'étude des Cokillajes, \& de les inviter a augmenter par de nouveles recherches le nombre des quarante-fix efpeces qu'il a reconu dans nos environs. Fait a Paris ce 12 Novembre 1766 .

$$
\text { Signé, A D AN SON. }
$$

\section{PRIVILEGE DU ROI.}

$\mathrm{I}$

OUIS, par la grace de Dieu, Roi de France \& de Navarre: A nos amés \& féaux Confeillers, les Gens tenant ros Cours de Parlement, Maîtres des Requêtes ordinaires de notre Hôtel, Grand-Confeil, Prévôt de Paris Bail!ifs, Sénéchaux, leurs Lientenans Civils, \& aurres nos Jufticiers qu'il appartiendra : S A L UT. Notre amé le Sieur vieffroi, Nous a fair 
expofer qu'il defireroit faire imptimer \& donner as Public un Ouvrage qui a pour titre: Traité fommaire des Cuquills, tant fuvizatles que terreftres, s'il Nous plaifoir lui accorder nns Lettres de Privilegés pour ce nécelfaires' A C ES C A U SES, vónlane favorablement traiter l'Expofant, Nous lui avons permis \& permetrons par ces préfentes de faire imptimer ledit Ouvrage autant de fois que bon lui femblera, de le faire vendre \& débiter par-tout notre Royaume, pendant le tems de fix années confécutives, à compter du jour de la date des Préfentes. Faifons défenfes à tous Imprimeuts, Libraires, st autres perfonnes de quelque qualité \&s condition qu'elles foient d'en introduire d'impreffion étrangere dans aucun lieu de notre obćiflance, comme aufis d'imprimer, faire imprimer, vendre, faire vendre, débiter ni contrefaite ledit Ouvrage, ni d'en faire aucun extraic fous quelque prétexte que ce puilíe être, fans la permifion exprefle \& par écrit dudic Expofant, ou de ceux qui aurone droit de lui, à peine de confifcation des Exemplaires contrefaits. de trois mille livres d'amende contre chacun des Contrevenaırts, dont un tiets à Nous, un tiers à 1 Hôtel..Dicu de Paris, Br l'autre tiers audit Expolant, ou à celui qui aura droit de lui, \& de tous dépe ds, dommages \& intérêts. A la charge que ces préfentes feront enregiftrées tour au long fur le Regiftre de la Communaucé des Imprimeurs \& Libraires de l'aris, danstrois mois de la date d'icelles: que l'impretion dudit Ouvrage fera faite dans notre Royaume \&x non aillelirs, en bon papier \& beaux caracteres, conformément aux kéglemens de la Librairie, \& notanment à celui du io Avril $x>2\{$, à peine de déchéance du présent Privilége, qu'avint de lexpofer en vente le manufcrit qui aura fervi de copie à l'impreffion dudir Ouvrage tera remis dans le même état où l'Approbarion aura été donnée, ès mains de notre très cher \& féal Chevalier Chancelier de France; le Sirue de Lamoigron, \& qu'sl en fera enfuire remis deux Exemplaires dan notie Bibliotheque publique, un dans celle de notre Château du Louvre, \& un dans celle dudit Sieıs 
ZAMOIGNON, \&: un dans celle de notre très cher $\&$ - féal Chevalier Vice-Chancelier \& Garde des Sceaux de France, Le Sieur de MEAU puU, le tout à reice de nulité des Irćfentes. Du contenu delquelles Vous mandruns \& enjoignons, de faire jouir ledit Exporant \& fes ayans caufes, pleinement \& paifitlement, fans fouftrir qu'il leur foit fait aucun trouble ou empêchement. Voulons quà la corie des Préfentes, qui fera imprimée tout au iong au commencement ou à la fin dudit Ouvrage, foir renue ponr duenent fignifiée, \& qu'aux copies collationn'es par l un de nos amées \&c f'́aux Confeillers Secrétaires, fni foit ajoutée comme à l'original. Commandons au premier notre Huisfier ou Sergent fur ce reguis, de faire pnur l'exécution d'icelles tous aetes requis ü néceftaires, fans demander autre remifiou, \& nonobftant clameur de Haro, Charte Nurmande, \&x Lettres à ce contraires. CAR tel eft notre plaifr. Eonné à Verfailles, le trente-unieme jour du nois de Décembre, l'an de grace mil fept cune Ioixante-fix, \& de notte Regne le cinquante-deuxieme. Jar le Roi cn fon Confeil.

Signé, LEBEG UE.

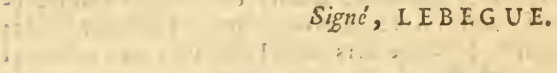

- Degifré fur le Regifre XII. de la. Chambre Roynle E Syndicale des Liliraires. E Imprimeurs de Paris, nn. $1288, f o l .8 \mathrm{~s}$. conformément au Réglement de 1723 , qut fair défenfes, artide 4I, à toutes perfonnes dequelques qual ris \& cond $t$ ors qu' clles foient autres que lis Libraures E. Im rimeurs, de vendie, aébiser, fai-e aficher atcuns lives pour les zendre on leurs nems; fo t qu'lls $s^{3} \in$ ne difent ies cuieurs ou autrement, Es à la charge de foucnir d'a fifóte Crambro neuf tximp'aires pecferics par -'article $10 \delta$ du même Reglemert. A I'aris ce: : Janvier 1767.

\section{GANEAU, Syndic.}




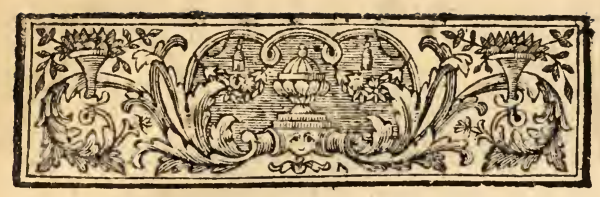

\section{T R A I T E SOMMAIRE}

\section{DES COQUILLES,}

tant fluviatiles que terreftres,

QUISE TROUVENT

\section{AUX ENVIRONS DE PARIS,}

I NTRODUCTION.

Tout le monde connoît fous le nom de Coquilles, ces efpeces de demeures dures, \& 


\section{2}

$$
\text { T R A I T E }
$$

comme pierreufes, qui renferment des animaux mous, fans os ni arrêtes \& fans articulations fenfibles, que les Naturaliftes ont rangés dans la claffe des Vers. Ces Coquilles ne font pas toutes de la même forme. Les unes ne font compofées que d'une feule piece, qui fouvent eft roulée en forme de fpirale; on les appelle Univalves. D'autres font compolées de déux pieces, ou efpeces de battans qui fe joignent l'un contre l'autre, \& renferment l'animal dans leur cavité; ce foncles Bivalves. Enfin, il yen a qui fon compofées d'un plus grand nombre dẹ 


\section{DES COQUILLE's ;}

pieces, \& auxquelles on a donné le nom de Multivalves. Toutes les efpeces de Coquilles font renfermées fous ces trois divifions. Quelques Naturaliftes en ont cependant ajouté une quatrieme; c'eft celle des Operculées.

Ces Coquilles ont été ainfi appellées, parceque leurouverture eft fermée par une petite plaque fur laquelle paroiftent des fpirales, \& $x$ qui eft tantôt de la nature de la Corne, tantôt de la même lubftance que la Coquil1c. Comme cette plaque a reçu le nom d'Opercule, on a donné aux Coquilles qui la portent le 


\section{T R A I T É}

nom de Coquilles operculécs. Ces Coquilles quoiqu'Univalves, femblent fe rapprocher des Bivalves par cette petite piece, qui eft comme une feconde Coquille. Il y a, fur-tout, quelques genres oi cet Opercule femble articulé avec la grande Coquille; en quoi ils refremblent encore plus aux Bivalves, dont les deux battans font articulés enfemble.

De ces Coquilles les unes font terreftres, les autres aquatiques, La mer fournit des Coquilles de toutes ces différentes divifions: mais parmi les Coquilles terreftres, nous ne connoiffons all- 


\section{DES COQUILLE.}

cunes Bivalves ni Multivalves; toutes font Univalves: il y a feulement quelques Operculées terreftres. Les Coquilles d'eau douce, les feules aquatiques dont nous avons à parler ici, nous fourniffent des Bivalves \& des Univalves, tant fimples qu'operculécs: mais jufqu'ici on n'en a trouvé aucune qui foit Multivalve. Ainfi nous nous contenterons de divifer les $\mathrm{Co}$ quilles que l'on trouve aux environs de Paris en deux Sections:

la premiere comprendra les Univalves, \& les Bivalves compoferont la feconde. 


\section{SECTION PREMIERE.}

COQUILLES UNIVALVES.

I Es Coquilles univalves ne font compofées que d'une feule piece, ou d'un feul morceau, comme nous venons de le dire; mais la conformation de cette piece eft différente. Dans les unes ce n'eft qu'une plaque, concave en dedans, convexe en deflus, \& fous la concavité de laquelle l'animal eft renfermé : c'eft ce que l'on voit dans l'Ancile, qui n'a aucunes fpirales. Dans d'autres, \&x c'ent le plus 


\section{Traité des Coquilles. 7}

grand nombre, la Coquille forme une efpece de tuyau conique roulé en fpirale autour d'un axe; de façon que la partie la plus étroite forme les fpirales du centre qui font plus petites, tandis que les plus grandes s'éloignent de ce centre, \& vont former à la fin louverture de la Coquille. Parmi ces Coquilles en ́́pirales, les unes ont leurs fpirales roulées concentriquement les uncs autour des autres, \& forment une efpece de difque applati, fans que la Coquille ait aucun fommet; c'eft ce que l'on voit dans les Planorbes. Les autres onzleurs fpirales quife courbent 


\section{T $\mathrm{T} A \perp$ T}

cn tournant \& montant obliquement de bas en haut; ce qui donne à la Coquille une figure conique qui fe termine par un fommet plus ou moins pointu: cette forme de Coquille eft très commune parmi les Univalves. Enfin, cette efpece de cône formé par les fpirales eft plus ou moins allongé, ce qui donne différentcs formes aux Coquilles. C'eft d'après ces conformations différentes, \& fur-tout d'après celles de l'ouverture de la Coquille, que la plûpart des $\mathrm{Na}$ turaliftes ont rangé les Coquillages. Ce moyen étoit d'autant plus commode, qu'il eft facile 


\section{DES COQUilles. ?}

de conferver les Coquilles, \& d'examiner les rapports de leur conformation. Cependant les animaux qui habitent ces Coquilles pouvoient fournir des caracteres d'autant plus furs que la Coquille n'eft proprement que l'habit \& la demeure de l'animal, \& que des Coquilles très différentes en apparence, peuvent renfermer des animaux d'un genre tout-à-fait femblable, comme on en verra des exemples. Mais, la difficulté d'examiner des animaux qui vivent dans l'eau, \& la plîpart dans la mer, a jufqu'ici empêché de tirer les caracteres des 
10 T R A I T E

Coquilles, des animaux qu'elles renferment. M. Adanfon eft le premier qui ait furmonté cet obftacle, qui paroiffoit invincible aux Naturaliftes. Cet illuftre Académicien nous a donné dans fon Hiftoire Naturelle du Sénégal, la figure \& les caracteres des Coquillages de ce Pays, tant terreftres, que de mer $\&$. d'eau douce. Cet immenfe travail jette un nouveau jour fur cette partie intéreffante du regneanimal. C'cft d'après les vucs dece favant Auteur, que j'ai entrepris un travail bien moins étendu, \& le feul que me permiflent les affaires qui me 


\section{DES COQUILLES. II}

fixent à Paris. J'ai tenté de ranger méthodiquement, par des caracteres tirés des animaux, le peu de Coquillages tant terreftres que fluviatiles qui fe trouvent ici. Ces animaux ne comprennent que quarante - fix efpeces qui foient venues à ma connoiflance. Je les ai rangées rous fept genres, dont cinq compofent la premiere Section, celle des Univalves. Puiffe cet effai, engager les Jeunes Gens qui herborifent aux environs de $\mathrm{Pa}$ ris, à le perfectionner par de zouvelles Obfervations!

Les Coquilles de cette preA vij 
I 2 T R A I T E

miere Section fe rapportent aus cinq genres fuivants.
I․ Le Limas.
I․ Cochlea.

4 Tentacules, dont 2 plus grands portent des yeux à leur extrémité.

Coquille univalve en Tefta univalvis, SpiSpirale.

\section{$2^{\circ} \cdot \operatorname{Le}$ Buccin.}

2 Tentacules plats en forme d'oreille.

Yeux placés à la bafe des tentacules du côté intérieur.

Coquille univalve en fpirale \& conique.

$3^{\circ}$. LePlanorbe.

2 Tentacules filiformes.

Yeux placés à la bafe des tentacules du côté intéricur.

coguille univalve en Tefraunivalvis, Ćpira:
Tentacula 4, duo majora oculifera ad apicem. ralis.

$2^{\circ}$. Buccinum.

Tentacula 2 plana as. riformia.

Oculi ad bafim inter. ne.

Tefta univalvis, ,piralis, conica.

$3^{\circ}$. Planorbis.

Tentacula 2 filiformia.

Oculi ad bafim interne. 
DES COQUILIES. I 3

Cpirale \& ordinai- lis, plærumquederement applatie. preila.

$$
4^{\circ} \text {. Le Nérite. } \quad 4^{\circ} \text {.Nerita. }
$$

2 Tentacules.

Tentacula 2.

Yeux placés à la bafe Oculi ad bafím ex: des tentacules du terne. côté extéricur.

Opercule à la Co- Operculumteftx. quille.

Coquille univalve en Tefta univalvis, $\mathrm{Cp}_{\mathrm{p}}$ fpirale \& prefque ralis, fubconica. conique.

$$
5^{\circ} \text {. L'Ancile. } \quad 5^{\circ} \text {. Ancrius. }
$$

2 Tentacules.

Tentacula 2.

Yeux placés à la bafe Oculi ad bafim inter. des tentacules du ne. côté intérieur.

Coquille univalve, Tefta univalvis, con. concave \& unie. cava, xqualis.

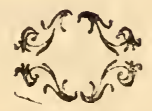




\section{Le Limas. Cochlea.}

4 Tentacules, dont 2 Tentacula 4 , duo ma. plus grandsportent jora oculifera ad des yeux à leur ex- apicem. trémité.

Coquille univalve en Tefta univalvis, Cpirpirale. ralis.

Famille premiere, à Familia prima, Tefta Coquille arrondie. fubrotunda.

- Seconde, à Co- - Secunda, Terta quille allongée. longa.

Les Limascompofent le genre le plus nombreux que nous connoiffions parmi les Coquilles de ce Pays-ci. De ce genre font les différentes efpeces que l'on rencontre dans les Jardins, les Vignes \& les Campagnes, \& qui font connues fous le nom de $\mathrm{Li}$ maçons. Tous ces animaux fone rerreftres, \& courent à terre ous 


\section{Traité des Coquilles. is}

fur les plantes, à l'exception d'une feule efpece que nous avons nomméel'A mphibie, parcequ'elle vit également fur la terre \& dans l'eau.

Les animaux qui vivent dans ces Coquilles, font du même genre que les Limaces qu'on trouve dans les Jardins \& les. Caves. Les uns \& les autres ont également quatre tentacules, dont deux font plus courts \& deux plus longs. C'eft à l'extrémité de ces derniers que font placés deux corps arrondis qui contiennent dans leur milieu une partie plus brune, \& qui paroiffent être les yeux de ces 
16 T R A I T E

animaux. La feule différence des Limas \& des Limaces, c'eft que les premiers ont une Coquille tournée en fpirale, dans laquelle ils peuvent fe retirer entierement, \& dont ils font fortir la partie antérieure \& inférieure de leur corps, lorfqu'ils veulent marcher, emportant leur Coquille avec eux; au lieu que les Limaces ont le corps nud \& fans Coquille à l'extérieur: il eft vrai qu'en les difféquant on trouve dans l'intérieur de leur Corps, vers la tête, une efpece de petir offelet long, mince \& applati, de la même fubrtance que les Coquilles; mais il n'en a point 


\section{DES COQUILLES. I7}

l'ufage \& ne paroît point à l'extérieur.

Les Limas font tous animaux hermaphrodites; ils ont tous les deux fexes, \& les parties font fituées au côté droit du col de l'animal, à l'endroit qui fort de la Coquille lorfque le Limass'allonge pour marcher. Mais quoique ces animaux aient les deux fexes, ils ne peuvent cependant engendrer feuls; ils s'accouplent toujours deux enfemble : feulement tous les deux font réciproquement l'office de mâle \& de femelle, enforte que l'accouplement entr'eux eft double.

Lorfque ces animaux veulent 


\section{8 \\ T R A I T E}

s'accoupler, ils commencent par un prélude íngulier : la nature les a pourvus d'une efpece de dard ou ficche à quatre aîles, d'une fubfance caflante, ferme \& affez femblable à celle de la Coquille. Cet aiguillon fort par la même ouverture du col qui donne iflue aux parties mâle \& femelle; \& lorfque ces animaux s'approchent, l'aiguillon de l'un pique l'autre, abandonne la partic d'oil il fort, \& tombe à terre ou refte attaché au Limaçon qui a été piqué : cclui-ci fe retire; mais bientôt après il fe rapproche, pique l'autre à fon tour, après quoil l'accouplement 


\section{DES COQuilles. I9}

s'exécute. Ces animaux s'accouplent jufqu'à trois fois de quinze. cn quinze jours, \&x chaque föis la nature fait les frais d'un nouvel aiguillon. Leurs accouplemens durent chacun plufieurs heures, \& pendant ce tems ils paroifent comme engourdis. Dix-huit jours environ après, les Limaçons rendent par la même ouverture du col, une grande quantité d'œufs blancs, revêtus d'une coque membraneufe, qui lorfqu'elle eft feche devient caffante, \& de la groffeur de la moitié d'un pois. Ils cachent ces œufs en terre, où jeles ai trouvés pluficurs fois. 
Tel eft l'accouplement des Limas. On verra cependant dans le détail quelques difiérences fuivant les cffeces:il y en a par exemple qui ont deux dards ou aiguillons vénériens, tandis que les autres n'en ont qu'un.

Les Limas vivent d'herbes \& de feuilles; ils font même fouvent de grand́s dégats dans les Jardins \& les Potagers: la nature les ayant pourvus de deux machoires dures, ofleufes \& tranchantes, avec lefquelles ils coupent \& brifent les feuilles.

Aux approches de l'hyver les Limas fe retirent dans quelques trous ou ils fe mettent à l'abri, 


\section{DES COQUILLES. 2 I}

\& ils ferment alors leurs Coquilles avec une efpece de couvercle blanc \& comme plâtreux, formé par leur bave ou mucofité, épaiflie. On les trouve fouvent ainfi fermés à la fin de l'hyver, jufqu'au mois de Mars, \& c c'eft alors que les Gens de la Campagne les ramaffent pour les manger. Ce couvercle plâtreux qui ferme l'ouverture de la Coquille, n'eft qu'une fimple plaque; il differe des opercules en ce que fur ceux-ci on apperçoit des fpirales qui ne fe voient point fur ce couvercle. D'ailleurs l'opercule eft une partie effentielle de l'animal qu'il con- 
22 T R A I T E

ferve en tout tems, avec laquelle il ferme fa Coquille toutes les fois qu'il le veut; au licu que ce couvercle plâtreux n'eft qu'une fimple concrétion étrangere à l'animal \& fans organifation. Auffi, dès le commencement du printemps le Limaçon rompt \& détruit ce couvercle; il fort alors de fa Coquille, va chercher fa nourriture \& renouveller fes dégats.

Nous avons divifé ce genre qui eft affez nombreux en deux familles, à raifon de la forme des Coquilles de ces animaux. Le premiere renferme ceux dont les Coquilles font arrondies, 


\section{DES COQUILIES. 23}

telles que, celles des Limaçons des Jardins. La feconde comprend ceux qui ont des Coquilles allongées \& comme en clocher. On peut fubdivifer cette feconde famille en deux ordres. Le premier eft compofé des $\mathrm{Li}_{\mathrm{i}}$ mas dont les volutes de la Coquille font contournées de gauche à droite, comme font les Limaçons \& la plus grande partie des Teftacés univalves. Les autres qui compoferont le fecond ordre, ont au contraire les volutes de leur Coquille tournées de droite à gauche; ce qui a fait appeller ces efpeces de Coquilles par plufieurs Naturaliftes 
$24 \quad T$ R A I T du nom très impropre d'Uniques, d'autant que dans beaucoup de genres de Coquilles de mer, on trouve de ces Coquilles uniques.

\section{§. I.}

\section{A Coguille arrondie.}

I. Cochlea, tefta utrinque convexa, rufefcente, quinque fpirarum.

Linn. Faun. Suec. 1293 . Cochlea, tefta ovata, quinque fpirarum, Pomatio dicta.

Linn. Syft. Nat. edit. 10, t. I, p.771, n. 593 . Helix, tefta umbilicata, fubovata, obtufa, decolori, apertura fubrotunda-lunata. Vulgò Pomatia. Gesn, 


\section{DES COQUILI'ES. 25}

Gefn. Aquat. 25s. Pomatia.

Aldrov. Exfang. 389. Cochlea terreftris, gyplo obferrata.

Lift. Angl. p. I I I, t. 2, f. I. Cochlea cinerea edulis, cujus apertura operculo craffo velut gypfeo per hye: mem clauditur.

Lifr. Exercit. Anat. I, p. I $6_{2}, t$. I.

Cochlea pomatia edulis Gefneri.

Lift. Hift. I, n. 46. Cochlea cinereorufefcens, fafciata, leviter umbilicata.

Dale, Pharmac. 394. Cochlea terreftris, Limax terreftris.

Merr. Pin. 207. Cochlea alba major cum fuo operculo.

Petiv. Mus. IV, n. I2. Cochlea alba major.

Swammerd. Bib. Nat. t. 4, f. 2.

Gualt. Teft. t. I, f. $A$. 
26 T R A I T É Argenville, Conchyl.part. I, tab. 28 ; f.r.

Idem, part. $2, t .9, f .4$.

Le Vigneron. Largeur is lignes.

Ce Limas eft le plus gros de ce Pays-ci. Sa coquille eft en fpirale, \& décrit quatre tours $\&$ demi $\&$ même près de cinq tours. Sa couleur eft un pcu fauve, avec quelques bandes plus foncées : le bord de fa bouche ou de fon ouverture eft peu faillant \& recourbé, \& fa coulcur eft la même que celle du refte de la Coquille. Pendant l'hyver cette bouche eft fermée par une efpece de couche plâ- 
DES COQUILLES. 27 treufe, blanche, tout-à-fait femblable à une Coquille d'œuf.

On trouve fouvent ce Limas dans les vignes; ce qui l'a fait apppeller le $V$ igneron. Pluficurs perfonnes le ramaffent dans les campagnes, fur-tout au printemps, lorfque fa coquille eft encore fermée, pour le faire cuire \& le manger. Son goût n'eft pas defagréable.

II. Cochlea, tefta utrinque conivexa, pullo maculata \& fafciata, quinque fpirarum, labro albo reflexo.

Liff. Angl. p. II 3. Cochlea major pulla maculata \& fafciata hortenfis.

$$
\text { Bij }
$$


28 T R A I T

Lift. Synop S. tab. $56, f .53$.

LE JARdinier. Largeur Io lignes.

Le Jardinier varie pour la grandeur; mais en général il eft au moins d'un bon tiers plus petit que le Vigneron. Cette Coquille a des bandes circulaires de taches brunes, cntrecoupées par des taches plus claires. Ce quil la fait aifément diftinguer des autres, c'ent que fon ouverture a un rebord faillant, d'un blanc laiteux en dedans. Cette ouverture fe ferme en hiver par le moyen d'une couche plâtreufe comme celle du Vigneron. 

DES COQUILLES. 2?

On trouve très fréquemment ce Limas dans les jardins, où il caufe beaucoup de defordre cn rongeant les plantes; c'eft ce qui l'a fait appeller le Jardinier. Quelques perfonnes le mangent comme le précédent; mais fa chair n'eft pas fi délicate. Ils font, l'un au défaut de l'autre, d'ufage en Médecine pour faire les bouillons \& le Syrop de Limaçons.

III. Cochlea, tefta utrinque convexa, flava, fufco fafciata, quinque fpirarum, labro furco refiexo.

Linn. Faun. Suec. I 294. Cochlea tefta utrinque convexa flava, fafcia fub$B$ iij 
30 T R A I T É

folitaria fufca, labro reflexo.

Limn. Syft. Nat.ed. I o, t. I , p.773, n. 604 . Helix tefta imperforata, fubrotunda, hevi, diaphana, fafciata, apertura fubrotundo lunata. V'ulgo Nemoralis.

Lif. Ang. I I 6, t. 2, f. 3. Cochlea citrina aut leucophra, non raro unicolor, interdum tamen unica, interdum etiam duabus aut tribus, aut quatuor, plærumque vero quinque fafciis pullis diftincta.

Idem, Hift. t. I, n. \$4. Cochlea interdum unicolor, interdum variegata, item variis fafciis depicta.

Swammerd, Bib. Nat. tom. I, t. 8, f. G. Cochlea hortenfis.

Merr. Pin. 207. Cochlea vulgaris, tefta variegata. 
DES COQUIILES. 3 ?

Petiv. Muf. 5, n. I 4. Cochlea vulgaris, tefta variegata.

Gualt. Teft.t. I, f.P.

Argenville, Conch. part. I, tab. 28 , f. 8.

Argenville, Corch. part. II, tab. 9, n. s. Cochlea femilunaris. Lift. Synop. Method. t. 57, f. 54 .

LA Livrée. Largeur ๆ, I o lignes.

Cette Coquille eft plus petite que les précédentes. Il en eft peu dont les couleurs varient autant : en général la couleur de la Coquille eft citronnée, lavée quelquefois d'un peu de rouge; mais tantôt la Coquille eft toute de cette couleur, fans aucune

$B$ iv 


\section{T R A I T É}

bande; tantôt elle eft chargéc d'une feule bande circulaire; d'autres fois de deux ou trois, quelquefois de cinq. Ces bandes brunes varient aufi pour leur grandeur \& leur pofition; mais l'ouverture de la Coquille a roujours un rebord affez faillant, de couleur brune, même dans celles qui n'ont aucune bande.

On trouve ce Limas par-tout dans les jardins \& les campagnes. Les bandes quile couvrent. \& lui donnent l'air d'une Livrée, l'ont fait appeller de ce nom.

IV. Cochlea, tefta utrinque con- 


\section{DES COQUIIIES. 33}

vexa alba, fex fpirarum, labro vix reflexo.

Lift. Anglo I 25, to $2, f$. I 2. Cochlea dilutè rufefcens, aut fubalbida, $f-$ nu ad umbilicum exiguo, circinato?

La Chartreuse. Diametre 6 lignes.

Cette Coquille peu élevée a environ un demi pouce de diametre, \& fa volute forme près de fix tours. Elle eft aifée à reconnoître par ce caractere \& par fa couleur toute blanche: l'animal qu'elle renferme eft pareillement blanc; auffil'a-t-on appellée la Chartreufe. On la trouve dans les bois; mais plus rarement que les précédentes.

\section{$\mathrm{Bv}$}




\section{4 \\ T R A I T É}

V. Cochlea, tefta utrinque convexa, fubtus perforata, ftriata, albido cinereoque fafciata, quinque fpirarum.

Argenville, Conch. part.11, t. $9, f .6$.

La grande Striée. Diamet. $s$ lig.

Sa couleur eft grife \& cendrée avec quelques bandes de taches plus foncées. En deffous, cette Coquille a un enfoncement ou ombilic, creux dans fon milieu: route la Coquille a des ftries longitudinales, fines; ce qui l'a fait nommer la Striée. On la trouve fréquemment dans les bois humides. L'animal que cette $\mathrm{Co}_{-}$ 
DES COQUILIES. 35 quille renferme, a une fingularité remarquable; c'eft qu'il eft pourvu de deux de ces dards, ou Spiculum Veneris, dont les Limaçons fe fervent \& qu'ils fe dardent mutuellement pour s'agacer, avant que de s'accoupler. Ces deux dards font dans deux capfules différentes. Tous les autres Limas, à l'exception dur grand Ruban, n'en ont qu'un feul, renfermé dans une feule capfule.

VI. Cochlea, tefta utrinque convexa, fubtus perforata, ftriata, alba, quatuor fpirarum, ore reflexo.

B vj 
36 T R.A I T É

'Argenville, Conch.part. II, t. $9, f_{.} \mathrm{T}$ La retite Stríke. Diametre I lig.

La couleur de cette petite Coquille eft blanche; elle eft chargée de quelques ftries longitudinales, difficiles à appercevoir à caufe de fa petiteffe: en deffous elle a un ombilic bien marqué, \& fon ouverture a un rebord faillant $\&$ très confidérable pourfa grandeur. Cette cfpece eft fort commune dans les bois, fous les pierres humides \& parmi les mouffes.

VII.Cochlea, tefta utrinque convexa, fubtus perforata, cor- 
DES COQUILIES. $3 \%$

nea, pellucida, nitida, quinque fpirarum.

Swammerd.Bibl.Nat. I, p. I $54 ; t a b .8$, f. 3. Minuta cochlea leviter depreffa.

Argenv. Conch. part. I, tab. 28 , fig. 4 . La Luisante. Diametre, lignes.

La Luifante eft ainfi nommée parcequ'elle eft très liffe. $\mathrm{SaCo}$ quille décrit cinq tours de volute: elle eft tranfparente $\&$ de couleur de corne claire, lorfqu'elle eft vuide; car du vivant de l'animal elle paroît d'un noir foncé, à caufe de la couleur du Limaçon qui eft très noir, \& que l'on voit à travers la 
38 T R A I T É

Coquille. En deffous elle a un ombilic creux. Elle fe trouve avec les précédentes, fous les pierres humides \& à l'ombre dans les bois.

VIII. Cochlea, tefta tota pellucida, fragili, fubvirefcente, utrinque convexa, f piris tribus.

Ia Transparente. Diamet. 2 lig.

Cette Coquille eft très liffe, luifante, convexe des deux côtés, nullement perforée en deffous, très mince, fragile \& $\operatorname{tranf}-$ parente comme un verre : elle a une teinte un peu verdâtre, \& elle décrit trois tours de fpirale, 


\section{DES COQuilizs, 39}

dont le premier eft fort grand; aufin fon ouverture eft-elle très large. On la trouve dans les mouffes humides, au bord des étangs; mais jamais dans l'eau où elle périt. C'eft même un moyen de tuer l'animal \& de le faire fortir de fa Coquille; ce qui ne fe pourroit faire autrement fans rifque de la caffer, à caufe de fon extrême délicateffe. Lorfque l'animal eft vivant, il a une appendice membraneufe avec laquelle il frotte $\&$ nétoye perpétuellement $f_{a}$ Coquille.

IX. Cochlea, tefta utrinque con- 
40 T R A I T É

vexa, fubtus concava, ftria-. ta, cornea, lineis tranfverfis ferrugineis, quinque fpiris rotundis.

Argenvil. Conchyl. part.II, t. 9, f. I 0.

Le Bouton. Diametre 2 lignes.

Cette petite Coquille eft très jolic. Sa forme eft affez applatic en deflus : en deflous elle eft plus convexe vers fes bords, avec un cnfoncement très confidérable à l'ombilic; ce qui la rend concave. Sa couleur eft pâle, femblable à celle de la corne; mais elle eft toute parfemée de taches tranfverfes rou- 

DES COQUILIES. $4 x$

geâtres, prefque à égale diftance les unes des autres; de plus toutc la Coquille eft chargée de ftries fines tranfverfes. Ces ftries \& ces taches font reffembler cette Coquille à un bouton joliment travaillé. On la trouve avec les précédentes dans la mouffe \& fous les pierres humides.

$X$. Cochlea, teftatutrinque convexa, fubtus perforata, limbo acuto, apertura ovata tranfverfa, fpiris quinque.

Linn. Faun. Suec. I 298. Cochlea tefta utrinque convexa, fubtus perfora- 


\section{4: T R A I T É}

ta, fpira acuta, apertura ovata tranlverfali.

Linn. Syft. Nat. edit. 1o, $\boldsymbol{I}, p .768$, n. 572 . Halix tefta carinata, umbilicata, utrinque convexa, apertura marginata tranfverfali ovata. Vulgo Lapicida.

Act. UpS. $1736, p .40$, n. 9. Cochlea tefta convexa, fubtus perforata, fpira acuta.

Pctiv. Muf. 69, n. 734. Planorbis terreftris Anglicus, umbilico minore, margine acuto.

Lift. Angl. I 26, t. 2 , f. I 4. Cochlea pulla, fylvatica, fpiris in aciem clepreffis.

Idem, Hift. I, p. 29, f. 62. Cochlea noftra umbilicata, pulla. 


\section{DES COQUILIES. 43}

La Lampe, ou le Planorbis terrestre. Diametre $5 \frac{1}{2}, 6$ lignes.

Cette Coquille eft une des plus fingulieres \& des plus rares de ce Pays-ci. Elle eft peu convexe en deffus, un peu plus en deffous, \&z percée d'un ombilic bien marqué. Elle décrit cinq tours de fpirale, dont l'extérieur eft très aigu, applati fur lcs bords, \&z coupé obliquement à l'ouverture; cnforte que cette ouverture eft prefque tranfverfale en deffous: cette bouche a des rebords blancs. Le relte de la Coquille a des ftries tranfverfes, \& eft de couleur pâle, 
44 T R A I T E

femblable à celle de la corne, tout parfemé de taches rougcâtres, affez grandes \& marquées; mais moins belles \& moins égales que dans le Bouton. On trouve cette Coquille, mais rarement, dans les bois autour de Paris.

XI. Cochlea, tefta utrinquic convexa, hifpida, fubtus perforata, fpiris quinque rotundatis, apertura ovata.

Linn. Faun. Suec. r 296. Cochlea, tefta utrinque convexa, hifpida, fpiris quinque rotundatis, fubtus perforata.

Linn. Syft. Nat. edit. 10, I, p.7, I, 


\section{DES COQUILLES. 45}

n. 59 r. Helix, terta umbilicata, convexa, hifpida, diaphana, anfractibus quinis, apertura fubrotundo-lunata. Vulgo Hifpida.

La Veloutée. Diametre 3 lignes.

Cette Coquille décrit cinq fpirales \& plus. Sa couleur eft femblable à celle de la corne, un peu brune. Le deffous forme un ombilic creux, bien marqué \&z fa bouche eft ovale, fans être bordée d'une levre faillante : mais ce qui la rend très reconnoiffable, c'eft qu'elle eft veloutée, ou parfemée de petits poils courts qui forment un duvet. On la trouve très communé- 
46 T R A I T É.

ment dans les bois humides \& dans les prairies.

XII. Cochlea, tefta fufca, hifpida, fupra plana, fubtus perforata, fpiris fex, a pertura triangulari, labro reflexo luteo.

LA Veloutée à bouche triangulaire. Diametre $4 \frac{1}{2}$ lignes.

Sa Coquille décrit fix fpirales: elle eft de couleur brune \& veloutée comme la précédente; mais platte en deffus \& même renfoncée dans fon milieu : en deffous elle eft percée d'un ombilic affez large. L'ouverture de fa bouche a un rebord ou une 


\section{DES COQUILIES. 47}

levre faillante de couleur jaunâtre, qui par fon contour rend. cette ouverture triangulaire. Cet ahimal eft affez rare. On le trouve quelquefois à Meudon, dans les endroits humides \& bas de ce Parc. Sa forme finguliere, \& qui approche de celle des Planorbis, l'a fait appeller par quelques perfonnes, le Planorbisterreftre.

\section{Cochlea, tefta alba, fupra} plana, fubtus finu amplo perforata, fpiris quinque, fafcia ferruginea.

Lift. Angl. p. I 26, tab.2, f. 13. Coch- 
$48 \quad T \quad R \quad A \quad I \quad T \quad \frac{1}{2}$

lea cinerea albidave, fafciata ericetorum.

Iegrand Ruban, ou Ruban piat. Diametre 6 lignes.

Le deffus de cette Coquille eft affez applati; mais le deffous a un large ombilic, qui laife voir les volutes en forme d'efcalier. La Coquille décrit fix fpirales : fa couleur eft toute blanche, à l'exception d'une bande de couleur fauve qui regne fui: le milieu des volutes, \& qui, affez ordinairement fur la derniere, eft accompagnée d'une feconde moins vive en couleur.

L'animal 
DES COQUILIES. 49

L'Animal de cette Coquille a deux dards vénériens de même que la Grande Striée. On peut voir ce que nous avons dit cideffus à ce fujet.

XIV. Cochlea, tefta alba, fupra plana, latere acuto, fubtus convexa, finu angufto perforata, Siris quatuor, fafcia fuprà unica, fubtùs plurimis fufcis.

Le petit Ruban, oll Ruban conVEXE. Diametre $2 \frac{1}{2}$ lignes.

Cette efpece eft plate en deffus, à peu près comme la précédente; en deffous elle eft con-

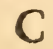


so T R A I T É

vexe \& perforée d'un ombilic étroit, en quoi elle en differe. Une autre différence; c'eft qu'elle ne décrit que quatre fpirales.

La forme plate du deflus fait que les fpirales ont un angle fur le côté vers le haut. Sa couleur eft blanche, avec une feule bande brune en deffus fur les volutes; mais en deffous, outre cette bande, il y en a quatre autres plus fines \& plus étroites.

On trouve en Normandie, dans les prés, au bord de la mer, une autre Coquille qui approche beaucoup de celle-ci, \&. qu'on pourroit nommer le $R u$ - 
DES Coquilis. SI ban marin; mais qui en differe en ce qu'elle a cinq fpirales \& qu'elle eft toute blanche en deffous, avec une feule bande brune en deffus.

\section{§. I I.}

A COCQUILLE ALLONGÉE.

I. à Volutes tournées à droite.

$X V$. Cochlea, tefta fulva obfcura, acuta, fpiris fex.

Lift. Angl. 122, tab. 2, f. 8. Buccinum rupium majufculum, circiter fenis orbibus circumvolutum. 'Argenv. Conchyl. part. I, t. $28, f$. I g.

C ij 
Le Grain d'Orge. Long. 3 lign:

Sa couleur imite celle de la Châtaigne; elle eft feulement un peu plus claire. Sa Coquille eft terne \& nullement brillante: elle décrit fix fpirales, \& a une ouverture ovale bordée d'une levre blanche.

Comme cette Coquille eft à peu près de la groffeur \& de la longueur d'un grain d'orge, on a tiré de cette reffemblance le nom qu'clle porte. On la trouve dans la mouffe \& fous les pierres humides.

XVI. Cochlea, tefta fufca, obfcura, acuta, fpiris octo. 


\section{DES COQUILLES 53}

Le Grain d'A voins. Long. 2 lign.

Ia couleur de cette Coquille eft brune \& nullement brillante. Elle décrit huit tours de fpirales. Son ouverture eft ovale, bordée d'une levre blanche, avec fept dents ou replis de même couleur, quatre en haut $\&$ trois en bas. Cette Coquille reffemble affez à la précédente; mais elle eft moins grande \& un peu plus pointue. On la trouve dans les mêmes endroits qu'elle.

XVII. Cochlea, tefta fulva, nitida, acuta, fpiris quinque.

Lif. Angl.pag. I 22, tab. $2, f .7$. Buc- 


\section{T R A I T É}

cinum exiguum, quinque anfractuum, mucrone acuto.

La Brillante. Longueur 2 lignes.

Cette Coquille approche de la précédente pour la coulcur, fi ce n'eft qu'elle eft plus pâle. Elle eft liffe \& brillante, \& ne décrit que cinq fpirales; en quoi il eft fort aifé de la diftinguer du. Grain d'Orge. Son ouverture eft ovale \& bordée d'une levre blanchâtre; mais peu marquée. Elle fe trouve dans les mouffes aquatiques au bord de l'eau; mais toujours fur terre, car, fi elle tombedans l'eau elle périt.

XVIII. Cochlea, tefta cinerea, 


\section{DES COQUILIES. IS}

acuta, ftriata, apertura quinque--dentata, labro reflexo, fpiris novem.

Argenv. Conchyl. 2, p. $8 \mathrm{I}$, t. $9, f_{.} 13$.

L'Anti-Nompareille. Long. 5 lign. Larg. I $\frac{1}{4}$ ligne.

Cette Coquille eft de coulcur cendrée, \& de forme allongée, aiguë par le bout. Elle a des fries fines longitudinales. Le bas de la Coquille fe reffere un peu : elle décrit neuf tours de fpirale. Sa bouche ovale a cinq replis ou dents, trois en haut \& deux en bas.

On trouve cette Coquille au pied des murs, \& dans les bois

$\mathrm{C}$ iv 
j6 T R A I T

parmi la mouffe. Nous l'avons appelléc Anti-Nompareille, parcequ'elle reflemble tout - à - fait à la Nompareille, dont nous parlerons tout à l'heure, n'en différant qu'en ce que fes volutes font tournées fuivant le fens ordinaire aux autres Coquilles, c'eft-à-dire de gauche à droite; au licu que celles de la Nompareille vont dans un fens oppofé, ou de droite à gauche.

XIX. Coçslca, tefta fubcylindracea obtufa, labro albo reflexo, rpiris octo.

Le Grand Barillet. Long. $2 \frac{r}{2}$ lign.

Quant à la couleur, cette 


\section{DES COQUILLES. 57}

Coquille approche de la couleur fauve, \& eft un peu tranfparente. Sa figure eft à-peu-près cylindrique, comme celle d'un petit tonneau ou baril, ce quil'a fait appeller Barillet, fes volutes formant comme les cercles d'un baril. Son fommet ne fe termine pas en pointe; mais il eft mouffe, obtus \& arrondi. On compte huit volutes fur cette Coquille, \& même prefque neuf. Son ouverture eft ovale, avec des rebords en forme de levres de couleur blanche, \& une arrête de même couleur, formée en feuiller, au milieu de l'ouverture. On la trouve parmi les

\section{$\mathrm{C} v$}


58

$T$ R A I T é

mouffes humides \& fous les pierres, dans les Jardins \& les Campagnes.

XX. Cochlea, tefta fubcylindracea obtufa, labro albo reflexo, fpiris fex.

Linn. Faun. Suec. I 301 . Cochlea, tefta fubpellucida, fpiris fex dextrorfis, fubcylindracea obtufa.

Linn. Syft. Nat. edit. I o, I p. 767. n. 568. Turbo, tefta turrita, obtufa, pellucida, anfractibus fecundis, apertura edentula. Vulgò Mufcorum.

Lif. Angl. ${ }_{21}$, t. 2, f. 6. Buccinum exiguum, flavum, mucrone obtufo, feu cylindraceum.

'1t. Celand. 99. Cochlea parva, fpiris feptem. 
DES COQUIL LES. 59 Argenvil. Conchyl.part. II, t. $9, f$. I I. Le Petit Bariliet. Long. x ligne.

Celle-ci reffemble en tout à la précédente, \& n'en differe que parcequ'elle n'a que fix fpirales, \& qu'elle eft plus petite de plus de moitié. On la trouve dans les mêmes endroits que le grand Barillet.

XXI. Cochlea, tefta alba, fragili, acuta, fpiris fex.

L'Aiguiliette. Longueur $1 \frac{2}{3}$ ligne, largeur $\frac{x}{4}$ ligne.

Cette petite Coquille eft longue, mince \& fine comme une aiguille; ce qui lui a fait donner

C vj 
60 T R I T $\frac{r}{E}$ le nom qu'clle porte. Elle eft blanche, fragile, délicate, \& elle décrit fix tours de fpirale. On la trouve fur les vieux murs entre les mouffes : il eft rare de la rencontrer avec l'Animal qu'elle contient; prefque toujours elle eft vuide.

\section{Cochlea, tefta membra-} nacea, fubflava, oblonga, mucrone obtufo, anfractibus tribus.

Linn. Faun. Suec. I 317.

Linn. Syft. Nat.edit. I o, I, pag. 776. n. 6I 4. Helix, tefta imperforata, ovata, obtufa, flava, apertura ovata. Vulgò $P$ urris. 


\section{DES COQUILIES. 6I}

Swammerd. Bibl. Nat. tom. I, p. I55,

t. $8, f .4$. Cochlea, figurx ovalis.

Liff. Angl. $4^{\circ}$, t. $2, f$. 24. Buccinum fubflavum, pellucidum, trium fpirarum.

Idem, Hift. Conchyl. 3, t. I $23, f .23$. Buccinum fubflavum, pellucidum, trium orbium.

Bonan. Recreat. $3, p$. II $9, f .54$.

Petiv. Muf. $8_{3}$, n. 808. Buccinum fluviatile noftras, tefta pretenui, fragili.

Tulp. Obferv. 200, t. 20I. Klein, Oftr. $t .3, f .70$.

Argenv. Conch. part. I, tab. 27, n.6, fg. ultima.

I'Amphibie ou l'Ambrée. Longueur 9 lignes, largeur $4 \frac{r}{2}$ lignes.

Les dimenfionsque nous don- 
62 T R A I T É nons de cet Animal, font prifes fur un des plus grands; il y en a de beaucoup plus petits. Sa Coquille eft mince, délicate, tranfparente; d'une coulcur très jaune \& ambrée, quand on en a tiré l'Animal qui eft noirâtre. On y apperçoit de petites ftries obliques, paralleles les unes aux autres. Elle forme feulement trois tours de fpirale, dont le premier eft très ample, le fecond moyen, \& celui d'en haut fort petit; ce qui fair que la pointe de cette Coquille eft obtufe, \& que fon ouverture eft large. Cette Coquille eft amphibie: on la trouve dans les 


\section{DES COQUILIES. 63}

étangs \& les ruiffeaux; mais fort fouvent elle en fort, \& grimpe fur les plantes voifines de l'eaụ. Elle eft très commune.

2. à Volutes tournées à gauche. XXIII. Cochlea, tefta fufca, opaca, apertura compreffa, labro albo reflexo, fpiris decem finiftrorfis.

Lif. Angl. p. $123, t .2$, f. х 0 . Buccinum pullum, opacum, ore compreffo, circiter denis fpiris faftigiatum.

Lift. Synops. Meth.t. 4I, f. 39 . Argenv. Conchyl. I, t. $28, f .19$. Argenv. Conchyl. II, p. 81, t.9j f. I4. 
64 TRAITE

La Nompareinde. Longueur 4 lign: largeur I ligne.

Sa Coquille eft allongéc; brune, opaque, \& nullement tranfparente. Vue de près, elle paroit avoir des ftries fines longitudinales. Le haut de la Coquille fe termine en pointe mouffe, le milicu eft plus renflé \& le bas fe refferre de nouveau. Elle fait dix tours de fpirale. Son ouverture eft oblongue, un peu refierrée, fur-tout vers le haut, \& elle eft bordée d'une levre blanche: au haut de louverture on apperçoit un repli ou une crête, pareillement 


\section{DEs CoQuilies. 65}

blanche. On trouve cette Coquille au pied des murs \& des vieux arbres, dans la mouffe $\&$ fur les pierres. Elle eft fort commune ici Sa formelui a fair donner le nom de Nompareille, fes volutes étant tournées dans un fens contraire à celui qui eft ordinaire aux autres Coquilles. C'ent par-là qu'elle differe de l'Anti-Nompareille, que nous avons décrite ci-deflus; ayant d'ailleurs dix tours de fpirale, au lieu que l'Anti-Nompareille n'en a que neuf.

XXIV. Cochlea, tefta fubcylindracea, obtufa, labro albo 
66

T R A I $T \dot{E}$

reflexo, ore quadridentato, fpiris octo finiftrorfis.

L'Anti-Barillet. Long. $3 \frac{1}{2}$ lignes, Larg. I $\frac{1}{3}$ ligne.

Cette Coquille eft de couleur jaunâtre, \& fon teft eft affez dur \& liffe. Elle eft prefque cylindrique, \& le haut fe termine en pointe très moufe, $\&$ à-peuprès comme le grand Barillet, auquel elle reffemble beaucoup. Elle décrit huit tours de fpirale. Sa bouche ovale eft un peu étranglée, a un rebord blanc affez épais, \& de plus, dans fon ouverture quatre replis ou dents blanches, dont une en haut, 
DES COQUIL LES. 67

deux à droite près l'une de l'autre, \& une plus groffe à gauche en regardant l'ouverture de face \& la pointe en haut. On trouve cette Coquille dans les mêmes endroits que la précédente. Comme elle reffemble au Barillet, mais que fes volutes font tournées dans un fens contraire, ou de droite à gauche; nous l'avons appellée l'Anti - Barillet. 


\section{Le Buccin. Buccinum.}

2 Tentacules plats en Tentaculaz plana au. forme d'oreille. riformia.

Yeux placés à la bafe Oculi ad bafim interdes tentacules du ne. côté intérieur.

Coquille univalve en Tefta univalvis, fpifpirale \& conique. ralis, conica.

Nows de Paris que trois efpeces de Buccins, qui toutes les trois font aquatiques, ne vivent que dans l'eau, \& périffent quelque tems après qu'on les en a tirées. Les Animaux que renferment ces Coquilles reffemblent beaucoup aux Limas; mais ils en different par des caracteres bien effentiels. Au licu que les Limas 


\section{Des CoQuilis. 69}

ont quatre tentacules ou efpeces de cornes à la tête, les Buccins n'en ont que deux, encore different-ils de ceux des Limaçons par leur forme : ils ne font point arrondis comme les leurs; au contraire, ils font larges \& applatis prefque commeles oreilles des Quadrupedes. On diroit que cet Animal a deux petites oreillesà fa tête. Une autre différence, c'eft que les yeux du Buccin ne font point pofés à l'extrémité des cornes, comme dans les Limaçons; mais au bas vers leur bafe, \& du côté intéricur de cette bafe. Si ce font, dans les uns $\&$ les autres, de véritables 
70 T R A I T É

yeux, comme on peut le croire, le Limas dont les yeux font élevés fur des efpeces de colonnes, doit mieux voir que le Buccin, qui porte fes yeux à la bale de fes tentacules, \& qui les ayant placés à la partic intérieure, doit encore être gêné par cette pofition: fes tentacules doivent fouvent lui cacher la vue des objets.

Les Buccins font Hermaphrodites, comme les Limaçons; maisleuraccouplement nes'exécute pas de même. Lorfqu'ils ne font que deux, l'accouplement n'eft point double; un feul fait l'office de mâle, \& l'autre celui 
DES COQUILLE. 71 de fémelle; ce qui vient de la pofition de leurs parties, qui rend le double accouplement impoffible: mais s'il en furvient un troifieme, alors il faifit celui des deux qui fait avec le premier l'office de mâle, s'accouple avec lui \& fait le même office; enforte que celui du milieu exercel'action de mâle \& de femelle; mais avec deux Buccins différens. Quelquefois on en voit dans les ruiffeaux, des bandes confidérables ainfi accouplées, dont tous font l'office de mâle $\&$ de fémelle avec deux de leurs voifins; tandis que les deux derniers, qui font aux deux extré- 
72 T R A I T É

mités de ce chapelet, moins fortunés que les autres, n'agiffent que comme mâle, ou comme femelle feulement.

Les Coquilles des Buccins, font toutes formées en fpirales \& allongées.

I. Buccinum, tefta oblonga; fufca, anfractibus fenis.

Linn. Faun. Suec. I 3 1 O. Cochlea, tefta producta, acuminata, opaca, anfractibus fenis, fubangulatis, apertura ovata.

Linn. Syft. Nat. edit. 1 o, I, p. 774 , n. 6I2. Helix, tefta imperforata, ovato-fubulata, fubangulata, apertura ovata. Vulgò Stagnalis.

Petiv. Muf. tab. 82, n. 805. Buccinum, 


\section{DES COQUILLES. $7 \hat{j}$}

num fluviatile noftras, oblongum, majus.

Lift. Angl. I 37, t. 2, f. 2 т. Buccinum longum, fex fpirarum, omnium \& maximum \& productius, fubtlavum, pellucidum, in tenue acumen ex amplifima bafi mucronatum.

Idem, Hift. Conch. 2, t. I 23, f. 2. Buccinum fubfavum, pellucidum, fex orbium, clavicula admodum tenui, productiore.

Frifch, Inj. 8, t. 7 .

Gualt. Teft. tab. s, f. r.

Aldrov. Teft. 3, p. 359, n. 3. Turbo

levis, in ftagnis degens.

Swammerd. Bibl. Nat. tab. $9, f .4$.

Le Grand Buccin. Long. I 4 lignes; largeur, lignes.

Cette Coquille, une des plus 
74 T R A I T É

grandes parmi les aquatiques des environs de Paris, eft de couleur brune, fouvent noirâtre; quelquefois claire, tranfparente \& ambrée; mais toujours d'une feule couleur. Sa forme allongée lui a fait donner le nom de Buccin, parcequ'elle reffemble aux Conques marines qui, fuivant la Fable, fervoient de trompettes aux Tritons. Elle décrit fix tours de fpirale, dont le premier, plus large que les autres, forme un ventre affez gros. Les autres vont en diminuant confidérablement, \& forment une pointe allongée \& très aiguë. Toute la Coquille a des 


\section{DES COQUILLE. 75}

ftries longitudinales peu fenfibles, \& de plus, chaque tour de fpirale a fouvent une raie longitudinale blanchâtre quila traverfe de haut en bas, \& qui femble faire la divifion d'un tour à l'autre. Cette Coquille eft très commune dans les ruiffeaux \& les étangs.

II. Buccinum, tefta oblonga, fufca, anfractibus quinque.

Lift. Angl. I 39, tab. 2 f. 22 . Buccinum minus, fufcum, fex fpirarum, ore anguftiore.

Petiv. Muf. 82, n. 306. Buccinum fluviatile noftras, oblongum, minus.

D ij 
76 T R A I T

Le Petit Buccin. Long. $3 \frac{1}{4}$ lignes, largeur I $\frac{2}{3}$ lignes.

Cette efpece approche beaucoup de la précédente; mais outre qu'elle eft quatre ou cinq fois plus petite, elle a encore plufieurs différences fenfibles. $x^{\circ}$. Elle n'a confamment que cinq tours de fpirale, au lieu de fix que marque Lifter; ce qui a pu induire en erreur M. Linnxus, quil'a confondue avec la précédente. $2^{\circ}$. Sa Coquille eft moins fragile \& moins mince que celle du grand Buccin. $3^{\circ}$. Elle eft moins allongée, à proportion, \& fa pointe eft 


\section{Des CoQuilies. 77}

moins aiguë; au contraire, le bas eft moins large, \& fa bouche par conféquent moins grande que dans la précédente. Ces différences fuffifent pour prouver quề ce Buccin n'eft pas le même que le Grand. On le trouve communément dans les ruiffeaux \& les étangs.

III. Buccinum, tefta diaphana, mucrone acuto breviffimo, apertura amplifima, anfractibus quatuor.

Linn. Faun. Suec. I 3 I S. Cochlea, tefta diaphana, anfractibus quatuor, mucrone acuto breviffimo, aperttira acutifima. 
$7^{8}$

T R A I T É

Linn. Syf. Nat. edit. 10, 1, p. 774, n. 61 7. Helix, tefta imperforata, ovata, obtufa, fpira acuta breviffima, apertura ampliata. Vulgò $A u$ ricularia.

Lift. Angl. $139, t .2, f_{0123}$. Buccinum pellucidum, flavum, quatuor $\mathrm{fpi}$ rarum, mucrone ampliffimo, teft $x$ apertura omnium maxima.

Idem, Hift. Conch. 2, t. I $23, f .32$. Buccinum fubflavum, pellucidum, quatuor orbium, ore amplifimo, mucrone acuto.

Idem, Exercit. 2, p. 54. Buccinum fluviatile, pellucidum, fubHavum, quatuor fpirarum, mucrone acuto, teft $x$ apertura patentiffina.

Petiv. Muf. $83, n .807$. Buccinum Aluviatile noftras, breve, ore parulo. 
DES COQuilies. 79

Argenv. Conch.part. I, tab. 27, n.7,

fig. 4 .

Idem, part. II, t. $8, f .6$.

Klein, Oftr. $54, t .3, f .69$.

Le Radix, ou Buccin ventru. Longueur 8,9 lignes, largeur 7 lignes.

Cette Coquille eft tranfparente \& affez fragile. Elle décrit quatre tours de fpirale, dont le dernier, ou celui d'en bas, eft prodigieufement gros \& large, $\&$ forme comme un ventre; ce qui rend l'ouverture de la Coquille très grande : les trois autres font très petits, $\&$ font une petite pointe aiguë, qui paroît comme entée fur ce gros ventre.

$\mathrm{D}$ iv 
80 T R A I T É

Les levres de l'ouverture fone un peu réfléchies en dehors. C'eft dans l'eau qu'on trouve ce Buccin avec les précédens; il eft un peu moins commun.

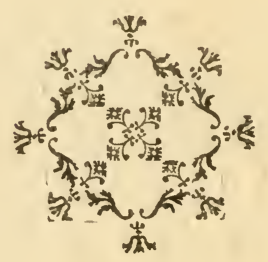




\section{LePlanorbe. Planorbis.}

2 Tentacules filifor- Tentacula 2 filiformes. mia.

Yeux placés à la bafe Oculi ad bafim interdes tentacules du ne: côté intéricur.

Coquille univalve en Tefta univalvis, fpirafpirale \& ordinai- lis, plarumquederement applatie. preffa.

Famille premiere, à Familia prima, Tefta Coquille applatie. plana depreffa.

- Secunde, a Co- -Secunda, Tefta ob. quille allongée. longa.

Troificme, à Cow - Tertia, Teltagloquille ovoïde. bofa.

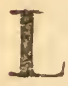

Es Planorbes, que quelques uns nomment Cornets de Saint Hubert, font des Coquillescompofées de plufieurs firirales, ordinairement applaties, comme les Cornes d'Ammon. Le carac. 
8 2 T R A I T E tere de ce genre eft aifé à faifir. Ces animaux n'ont que deux tentacules, comme les Buccins, \& leurs yeux font placés à la bafe de ces tentacules, du côté intérieur, comme dans ces Animaux; mais les Planorbes different des Buccins par un autre caractere; c'eft la forme des tentacules. Ceux des Buccins, ainfi que nous l'avons dit, font larges \& applatis, comme des oreilles; au lieu que ceux de ce genre font minces, arrondis \& filiformes. C'eft par ce dernier caractere qu'on diftingue ces deux genres. La forme de la Coquille peut aufliy entrer pour quelque chofe. 
DES COQUIILES. 83

En général elles font ordinairement applaties; \& ce font celles qui compofent la premiere famille. Cependant cette forme de Coquille n'eft pas tellement effentielle aux Animaux de ce genre, qu'il n'y en ait de figure très différente. Nous en connoiffons deux, dont l'un a une Coquille de figure allongée en forme de vis, \& dont nous avons fait la feconde famille; \& l'autre, en porte une globuleufe $\&$ arrondie comme un ceuf; c'ent celui de la troifieme famille. Ces deux Animaux, malgré la différence de leurs Coquilles, fe

$\mathrm{Dvj}$ 
84 T R A I T É

rapportent à ce genre; ils en ont les caraceres.

Tousles Pianorbes font aquatiques, \& ne vivent que dans l'eau. Ces Animaux font Hermaphroditcs, \& leur accouplement eft parfaitement femblable à celui des Buccins; ainfi nous ne répéterons pas ce que nous avons dit ci-deffus à ce fujet. On peut confulter l'article des Buccins.

\section{§. I.}

T'A COQUILLE APPLATIE.

1. Planorbis, tefta plana, pulla, 
DES COQUILLES, \&S

fupra umbilicata, anfractibus quatuor teretibus.

Linn. Faun. Suec. 1304. Cochlea, tefta plana, pulla, fupra umbilicata, anfractibus quatuor teretibus. .

Linn. Syf. Nat. edit. Io, I, p. 770 , n. 587 . Helix, tefta fupra umbilicata, plan, nigricans, anfractibus quatuor teretibus. Vulgo Cornea.

Liff. Angl. 143 , t. 2, f. 26 . Cochlea, pulla, ex utraque parte circa umbilicum cava.

Idem, Exercit. 2, p. 59. Purpura feu cochlea fluviatilis, major, compreffa.

Gualt. Teft. t. 4, f. $D D$.

Argenville, Conch.part. II, $t_{0} 8, f_{0} 7_{\text {. }}$ 
Le Grand Planorbe à fpirales rondes. Diametre 8 lignes.

Cette Coquille décrit quatre tours de volute, qui ne s'élevent point en fprrale, comme les autres genres de Coquilles; mais qui tournent autour d'eux-mêmes, \& s'enveloppent comme la plupart des efpeces de ce genre. Ces volutes font cylindriques; ce qui rend les bords de la Coquille ronds. Son teft eft de couleur obfcure, un peu tranfparent, légerement ftrié, fouvent couvert d'une efpece de boue, un peu luifant lorfqu'il eft nétoyé. La Coquille eft prefque 
DES COQUILIES. 87 plate en deffous, comme les Cornes d'Ammon; en deffus, elle eft concave, \& forme un ombilic très creux. On la trouve communément dans les petits ruiffeaux \& les étangs. L'Animal qu'elle renferme eft d'une couleur fort noire, \& fi on ouvre fon corps, il en fort une liqueur d'un rouge foncé.

II. Planorbis, tefta plana, alba, utrinque concava, anfractibus quinque teretibus.

Linn. Faun. Suec. 1305 . Cochlea, tefta plana, alba, utrinque concava, anfractibus quinque teretibus.

Linn. Syft. Nat. edit. 10, $1, p .770$, 
88

R A I I T

n. 588 . Helix, tefta utrinque concava, plana, albida, anfractibus quinque teretibus. Vulgo Spirorbis

Act. UPS. $1736, p .40$, n. 2. Cochlea, tefta depreffa, utrinque fubrquali, fpira tereti.

Le petit Planorbe, à cinq fpirales rondes. Diametre $\mathrm{I} \frac{1}{2}$ ligne.

La couleur de cette efpece de Coquille eft blanchâtre. Elle eft plate, un peu concave tant en deffus qu'en deffous, \& elle décrit cinq tours de fpirale, qu'on apperçoit également des deux côtés. Ses fpirales font arrondies, ainfi que fon ouverture. On la trouve dans les étangs. 


\section{DES COQUILIES. 89}

III. Planorbis, tefta fufca, fupra plana, fubtus concava, perforata, anfractibus fex teretibus.

Le petit Planorbe, à fix fpirales rondes. Diametre $\mathrm{I} \frac{1}{4}$ ligne.

Cette petite efpece eft plate en deffus, concave en deffous, avec un ombilic enfoncé \& $x$ perforé au milieu; de façon qu'on ne voit gueres que deux tours de fpirale en deffous, qui paroiffent affez larges; mais en deffus, on en compte fix fort ferrés. Ces fpirales font arrondies comme celles des deux efpeces pré- . cédentes, fans arrête ni rebord, 
90

T R A I T É

\& l'ouverture bien perpendiculaire forme une efpece de lunule ou de croiffant. Cette Coquille eft de couleur brune: on la trouve dans l'eau avec les autres Planorbes; mais elle eft un peu rare.

IV. Planorbis, tefta plana, fufca, fupra concava, anfractibus quatuor, margine promirulo

Linn Faun. Suec. 1306. Cochlea, tefta plana, fufca, fupra concava, anfractibus quatuor, margine prominulo.

Linn. Syft. Nat. edit. 10, I, p. 76), n. 578 . Helix, tefta fubcarinata, umbilicata, plana, fupra concava, 


\section{DES COQUILLES. 9I}

apertura oblique ovata, utrinque acuta. Vulgo Planorbis.

Lift. Angl. 1 45, t. 2, f. 27. Cochlea, fufca altera parte planior, \& limbo infignita, quatuor fpirarum. Idem, Hift. Conch. II, t. I $38, f .42$.

Cochlea, fufca, limbo circumfcripta.

Petiv. Gafop. 16, t. 10, f. 1 1. Planorbis minor fluviatilis, acie acuta. Gualı. Tejl. t. 4, f. EE. Klein, Oftr. t. x, f. 8.

Le Planorbe, à quatre fpirales à arrête. Diametre 6 lignes.

Cette Coquille eft applatie \& un peu renfoncée dans fon milieu, tant en deffus qu'en deffous. Elle eft noire lorfque l'Ani- 
92 T $R$ A A I

mal eft vivant; mais lorfqu'il a été tiré de fa Coquille elle eft tranfparente, de couleur de corne, avec de petites ftries qui tra-verfent les fpirales obliquement. Les tours de fpirale que décrit la Coquille font au nombre de quatre, \& quelquefois de cinq, dont celui du milieu eft très petit, \& fouvent incomplet. $\mathrm{La}$ fpirale extérieure a dans fon milieu une arrête ou bord aigu, qui regne tout autour de la Coquille. L'ouverture ou la bouche eft ovale, un peu aiguë par les deux bouts, \& regarde obliquement le deffous, ayant fon bord fupérieur plus long que l'infé- 


\section{DES COQUILLES. 93}

rieur. On trouve cette Coquille dans les marais, les étangs \& les rivieres.

V. Planorbis, tefta plana, fufca, fupra concava, anfractibus fex, margine asuto.

Linn. Faun. Suec I 307 . Cochlea, tefta furfa, plana, fupra concava, anfractibus quinque, margine acuto. Linn. Syf. Nat. edit. 10, 1, p. 770 , n. 583 . Helix, tefta carinata, plana, fupra concava, apertura ovali. Vulgo Vorcex.

Lift. Angl. x 45, to 2, f. 29. Cochlea exigua fubfufca, altera parte planior, fine limbo, quinque firiarum. Gualt. Teft. t. 4, f. GG. 
94 . T R A I T E

Le Planorbe, à fix fpirales à arrête. Diametre 3 lignes.

Cette ef́pece reffemble beaucoup à la précédente pour la forme \& pour la couleur; mais outre qu'elle eft plus petite, elle eft moinôtriée, \& a plus de tours de fpirale : ordinairement fix. De plus, l'arrête de la fpirale extérieure eft moins au milieu que dans l'efpece ci-deffus, \& forme le bord inférieur fur lequel la Coquille eft appuyée. On trouve ce Planorbe avec les précédens.

VI. Planorbis, tefta plana, fubtus concava, anfractibus tribus deorfum marginatis. 


\section{DES COQUILLES. 95}

Linn. Faun. Suec. I 308 . Cochlea, tefta plana, fupra convexa, fubtus concava, anfractibus quatuor deor-fum marginatis.

Le Planorbe, à trois fpirales à arrête. Diametre 2 lignes.

Celle-cieft encore de la même forme \& đacla même couleur que les efpeces précédentes; mais elle eft plus petite, \& fes fpirales au nombre de trois, ou trois $\&$ demi, font beaucoup plus groffes. Le deffus \& le deffous de la Coquille font un peu concaves. La dernicre fpirale, ou le bord extérieur a une arrête faillante $\&$ aiguë, placée tout-à-fait à la 
96 T R A I T

partie inférieure; ce qui rend ce côté des fpirnles plait. On trouve cette Coquille avec les précédentes.

VII. Planorbis, tefta plana, fubvillofa, fubzus concava, anfractibus tribus in medio marginatis.

Le Planorbe veloutti. Diametre 2 lignes.

Ce petit Planorbe décrit trois tours de f pirale. Il eft plat en deffus \& concave en deffous; chargé de ftries légeres, longitudinales \& tranfverfes. Sa fpirale extéricure a un rebord ou une arrête, mais placéc dans fon milieu, 


\section{DES COQUILLES. 97}

lieu, \& non au rebord, comme dans la précédente. Cette fpirale extéricure eft plus groffe que les detrx autres, qui font fort petites. L'ouverture eft ovale \& placée obliquement, regardant le côté inférieur. Mais une fingularité de cette Coquille, c'eft d'être un peu velue, \& garnie d'un duvet de poils courts; ce qui fait qu'elle n'eft jamais polie ni brillantc. Elle a été trouvée dans l'eau avec les précédentes.

VIII. Planorbis, tefta plana, fubtus concava, anfractibus tribus, plicis tranfverfis fimbriatis.

E 
$98 \quad T$ R A I T E

Rofel.Inf.tom. 3 , tab. 97 , fig. 6, 7:

Le Planorbis tuilé. Diamet. $2 \frac{1}{2}$ lig.

Sa Coquille eft tranfparente, de couleur pâle, femblable à celle de la corne. Elle eft plate en deffus, concave en deffous : elle décrit trois tours de fpirale, dont l'extérieur eft beaucoup plus grand que les autres, \& a des ftries tranfverfes élevées, repréfentant des efpeces de feuillets allongés, plus longs vers le bord de la Coquille, \& un peu couchés; de façon qu'ils reffemblent à des tuiles couchées les unes fur les autres. Cette Coquille eft rare; on la trouve dans Ia petite riviere des Gobélins. 
DES COQUILIES. 99

\section{§. I I.}

A COQUille ALLONGÉE.

IX. Planorbis, tefta nigricante, producta, oblonga, anfractibus feptem, quadratis marginatis.

Argenv. Conch. pare. II, pl.8, fig. 4.

Le Planorbis en vis. Long. 2 lignes; largeur $\frac{2}{3}$ lign.

Cette rare \& finguliere efpece eft de couleur noire. Ses fpirales pofées les unes au-deffus des autres la font reffembler à une vis. Ces fpirales, 'au nombre de fept,

Eij 
100 T R A I T É

font quarrées, \& ont à leurs bords tant fupéricur qu'inférieur, des angles bien marqués. Le total de la Coquille paroît un peu irrégulier, quoique les fpirales diminucnt également; parceque quelques-unes, furtout les deux petites d'en-haut, ne font pas pofées abfolument d'aplomb fur les autres. La Coquille eft percée en deflous d'un petit ombilic, \& fon ouverture cft oblique, bordée d'un peu de blanc.

Ce Planorbis n'a été trouvé ici qu'une feule fois dans la riviere des Gobelins, par M. de Juficu, qui m'a permis d'en 


\section{Des Coguiles. ror}

prendre ta figure \& la defcription; \& c'eft d'après le deffein que j'en avois fait, que feu M. d'Argenville l'a fait graver dans fon Ouvrage. La figure de l'Animal qu'il y a fait ajouter a été faitc d'idée.

\section{§. I I I.}

A COQUILLE OVOIDE.

$X$. Planorbis, tefta fragili, pellucida, globofa, anfractibus quatuor finiftrorfis.

Lift. Hift. Conch. t. I34. f. 34. Buccinum fluviatile, à dextra finiftrorfum tortile, triumque orbium, five neritodes.

Eiij 
102 T R A I T É

Lift. Angl. $142, t .2, f .25$. Buccinum exiguum, trium fpirarum à finiftra in dextram convolutarum.

Adanfon, Seneg. I, p. 7. Bulin.

Ia Bulle aquatique. Long. 2 lig. larg. I $\frac{1}{2}$ ligne.

La forme de cette efpece s'éloigne encore plus de la figure des autres Planorbis que la précédente: elle reffemble à un œuf. Ses fpirales font au nombre de quatre; mais celled'en bas, beaucoup plus grofle, fait prefque à elle feule le corps de la Coquillc. Les trois autres, pofées fur cette premiere, font très petites. Le teft de cette Coquille eft mince 


\section{DES COQUILLES. 103}

\& tranfparent, \& paroît noirâtre quand l'animal eft vivant, à caufe de la couleur noire de fon corps. Une autre fingularité de cette Coquille, c'eft qu'elle eft du nombre des uniques, où de celles dont les fpirales font tournées dans un fens contraire à celui des autres Coquilles, c'eft-à-dire de droite à gauche. Lorfque l'A nimal eft vivant, en marchant il fait fortir de fa Coquille une membrane, ou pellicule dentelée par les bords, qui couvre les trois quarts de cette Coquille. Nous l'avons appellée la Bulle, à caufe de fa forme arrondie, \& de fa tranfparence

$\mathrm{E}$ i $\nabla$ 
ro4 $T$ R A I T É.

- qui la fait reffembler à une bulle d'eau. Elle varic pour la grandeur : il y en a qui font plus groffes que les autres prefque du double. On trouve cette Bulle très communément danslesruiffaux \& les mares des environs de Paris. 


\section{Le Nérite. Nerita.}

2 Tentacules,

Yeux placés à la bafe des tentacules du côté extéricur.

Opercule à la Co- Operculumteftx. quille.

Coquille univalve en fpirale \& prefque colique.

\section{Tentacula 2.}

Oculi ad bafim externe.

Tefta univalvis, fpi. ralis, fubconica.

7

1

E s Nérites font trutes aquatiques, à l'exception de la premiere efpece, l'Elégante ftriée qui eft terreftre. Ces Animaux ne font point hermaphrodites comme les Limas, les Buccins \& les Planorbes, dont nous avons parlé jufqu'à préfent; ils font diftingués par le fexe: les uns font mâles, \& les autres femelles. Leur caractere eft d'avoir deux

$$
\text { Ev }
$$


I06 T R A I T É

tentacules; en quoi ils different des Limas qui en ont quatre : \& deux yeuxà la bafe de ces tentacules, mais au côté extérieur; ce quiles diftingue des Buccins \& des Planorbes qui les ont au côté intérieur. Un autre caractere bien effentiel de ce genre, c'eft d'avoir un opercule, ou petite lame de la nature du teft, furlaquelle on diftingue les empreintes d'efpeces de fpirales, \& qui fert à fermer exactement la Coquille. Ce caractere femble rapprocher ce genre de Coquilles Univalves des Bivalves, comme l'a très bien remarqué M.Adanfon. Quoique cet oper- 


\section{DES COQUIILES. 107}

cule foit retiré, \& ferme la Coquille, la partie du mâle paroît toujours un peu près du col à l'extérieur ; excepté cependant dans la Vivipare, où cette partie fe cache \& s'enfonce dans un des tentacules; en forte que les mâles de cette efpece, ont une de ces cornes plus groffe que l'autre; ce qui les fair diftinguer de leurs femelles à la premicre infpection. 'Toutes ces Nérites font ovipares, \&z pondent des œufs, à l'exception de la feule efpece que nous avons appelléc la Vivipare, parcequ'elle fait des petits tout vivants, qui fortent du corps de la mere, avec

$$
\text { Evj }
$$


108 T R A I T E

leurs petites Coquilles. On verra dans le détail des efpeces ce que chacune d'elles a de plus remarquable, la belle panache du Porte-Plumet, \& les jolies couleurs de la Nérite des Rivieres.

I. Nerita, tefta oblonga, cinerea, denfifime ftriata, maculis rufefcentibus, anfractibus quinque.

'Lift. Angl.p. I I g, tab. 2, f. . . Cochlea cinerea, interdum leviter rufefcens, ftriata, operculo teftaceo cochleato donata.

Colum. Purpur. cap. 9, p. I 8. Cochlea terreftris, turbinata \& ftriata.

Argenvil. Conchyl. part. I, t. $28, f .12$. Idem; part. II, t. $g, f . g$. 


\section{DES COQUILLS. IOG}

L'Elégante striée. Long. S lignes, larg. 4 lignes.

Cette Coquille eft allongée cn pyramide, dont la bafe eft large. Elle décrit cinq tours de fpirale, dont les deux d'en haut font fort petits. On remarque qu'clle eft couverte à l'extérieur deftries tranfverfes, fort ferrées, entrecoupées de quelques autres longitudinales. Sa couleur eit cendrée, variée de taches brunes, rougeâtres, oblongues, qui forment des raies tranfverfes; mais quand l'Animal eft mort, \& que la Coquille eft reftée vuide quelque tems fur la terre, ces 


\section{T R A I T É}

taches s'effacent, \& elle paroît toute de couleur cendrée. Les fries font auffi quelquefois plus ou moins marquées. L'ouverture de la Coquille eft prefque ronde, fans levres ni rebord, \& l'opercule qui la ferme eft en volute.

On trouvecette Coquilledans les bois humides; c'eft la feule de ce genre, qui ne foit point aquatique. L'élégance de fes ftries lui a fait donner, d'après Lifter, le nom qu'clle porte.

II. Nérita, tefta oblonga, fubviridefcente, fafciis tribus lividis, anfractibus quinque. 


\section{DES COQUILLE. IIJ}

Linn.Faun. Suec. I 3 I 2. Cochlea, tefta oblongiufcula, obtufa, anfractibus teretibus, lineis tribus lividis.

Linn. Syft. Nat. edit. 10, $1, p .772 . n$. 603 . Helix, tefta imperforata, fubovata, obtufa cornea, cingulis furcatis, apertura fuborbiculari.

Lift. Angl.p. $133, t .2, f .17$. Cochlea. maxima fufca, feu nigricans, fafciata.

Idem, Hift. Conch. II, t. $26, f .26$. Cochlea vivipara, fafciata.

Idem, Exercit. II, $p .17, t .2$. Cochlea maxima viridefcens, fafciata, vivipara.

Swammerd. Bib. Nat. 2. 9, f. 3. Cochlea vivipara.

Petiv. Muf. 84, n. 8 I 4 . Cochlea flur. viarilis, vivipara, londinenfis. 


\section{T R A I T Ét}

Gualt. Teft.t.s, f. I.

Aat. Upf: $1736, \rho .40$ n. 14 . Cochlea, tefta producto convexa Altviatilis.

Argenv. Conchyl. II part. pl. \&, f. 2.

La Vivipare, à bandes. Long. 8 lig. largeur 7 lignes.

La forme de cette Coquille eft femblable à celle de la précédente, à la grandeur près; car elle eft beaucoup plus grande: de plus, elle n'a que quelques fries longitudinales, peu apparentes, \& du refte, elle eft affez liffe. Sa couleur eft pâle un peu verdâtre; quelquefois brune, avec trois bandes d'un brun obcur, paralleles l'une à l'autre, 
DES COQUILIES. II

qui fuivent la direction des fpirales.Quand l'animal eft vivant, la Coquille eft plus brune, \& les bandes paroiffent moins que quand la Coquille eft vuide. Son ouverture eft ronde, fans rebord. ni levres, \& elle eft fermée par un opercule à volutes, comme dans l'efpece précédente.

Cette Coquille eft vivipare, au lieu que les autres de ce genre font ovipares; \& c'eft de la que lui a été donné le nom qu'elle porte. On la trouve dans les étangs \& les rivieres; il y en a beaucoup dans la Seine.

III. Nerita, tefta oblonga, pel- 
$114 T R$ A I T E lucida, cornca, anfractibus quinque.

'Linn. Faun. Suec. I 3 3. Cochlea, tefta oblonga, obtufa, anfractibus quatuor, laxis, cinereis, opacis, apertura fubovata.

Linn. Syft. Nat.edit. 1 o, I, p. 774 ; n. 6I6. Helix, tefta imperforara, ovata, obtufa, impura, apertura fubovata. Vulgo Tentaculata.

Zift. Angl. 135, tab. 2, f. 1 g. Cochlea parva fublava, intra quinque fpiras finita.

Act. UpS. $173^{6}, p .41$, n. 16. Cochlea palutris, teftr hiatu rotundo, contracto, fpiris laxis.

La petite Operculée aquatiquz. Long. $3 \frac{1}{2}$ lig. larg. $2 \frac{1}{2}$ lig.

On retrouve encoredanscette 


\section{DES COQUILLS. IIS}

Coquille la même forme que dans les deux précédentes. Son teft eft fragile, jaunâtre, tranfparent, femblable à de la corne, affez liffe \& fans ftries. Souvent elle eft couverte de limon qui la rend raboteufe, \& de couleur cendrée. Elle a cinq tours de fpirale, comme les précédentes, \& fon ouverture prefque ronde eft fermée par un opercule femblable aux leurs. On la trouve dans les rivieres $\&$ les eaux dormantes.

IV. Nerita, tefta ovata, livida pellucida, fubtus perforata, anfractibus tribus. 
II 6 T \& A I T E

Le Porte-Plumet. Longueur I lig. larg. I $\frac{1}{2}$ ligne.

Je ne trouve décrite nulle part cette efpece, l'une des plus fingulieres \& des plus jolies de ce genre, \& même de toutes celles que nous avons dans ce Pays-ci. Sa Coquille eft peu élevée, fort large, de conleur obfcure \& tranfparente. Elle ne décrit que trois tours de fpirale, \& en deffous elle eft perforée dans fon milieu par un petit trou. Son ouverture eft large pour fa grandeur, ix elle eft fermée d'un operculc à volutes. Le teft de liCoquille n'a rien, comme on 
DES COQUILLES. II 7

le voit, de bien fingulier. Mais fi on obferve l'Animal vivant, \& qu'on le voie fe promener dans un bocal plein d'eau; on apperçoit uutre les deux tentacules de la tête, qui lui font communs avec les Animaux de ce genre, \& avec plufieurs autres, un troifieme tentacule latéral, qui ne part point de la tête, comme les précédens, mais de côté, \& qui eft beaucoup plus long $\&$ plus fin. L'Animal le porte en l'air \& le remue. De plus, il a fur le côté droit de la tête un grand panache, ou efpece de Plumet, plus long que fes tenracules, qui a des deux côtés 
a IS T R A I T É

des barbes ondées. (Crifta pennata pennis undulatis.) Ce font les branchies de cet Animal, qui lui fervent au même ufage que celles des poiflons; je veux dire à refpirer. Rien n'eft plus joli que ce panache qui s'étend \& ic refferre, \& que cette Coquille porte comme un bouquet, fur le côté de la tête. C'eft à caufe de ce beau panache, que nous l'avons nommée PortePlumet. On la trouve dans les eaux des étangs, \& des petites rivieres. Elle eft commune dans la riviere des Gobelins.

V. Nerita, tefta lata, compacta, 


\section{DES COQUILIES. II}

fcabra, e cœruleo virefcente, apertura femi-ovata, anfractibus duobus.

Linn. Faun. Suec. I 3 18. Cochlea, nerita fluviatilis dicta.

Linn. Syf. Nat. edit. 1 O, I, p. 777 ; n. 632 . Nerita, tefta rugora, labiis edentulis. Vulgo Fluviatilis.

Lif. Angl. I 36, t. $2, f$. 20. Nerita fluviatilis è crruleo virefcens, maculatus, operculo fubrufo, lunato $\&$ aculeato donatus.

Idem, Hift. Conch. II, p. I, f. 38 . Nomen idem.

Petiv. Muf. $67, p .718$. Nerita thamenfis, exiguus, reticulate variegatus. .

Argenv. Conchyl. $1, t .27, f .3$. Idem , II, t. $8, f .3$. 
$120-\mathrm{T} R$ A I T É

La Nérite des Rivieres. Hauteur 2 lign. larg. s lign.

Prefque tout le monde connoît cetre Coquille, que l'on trouve très communément dans le ́able des Jardins, avec lequel elle a été apportée de la riviere. $\mathrm{Sa}$ forme cit très large \& peu élevée. Elle ne décrit que deux tours de fpirale; l'un fort large \& l'autre très petit, formant un petit ceil. Son ouverture eft en demi-cercle, fermée par un opercule de même forme. Le tcit de la Coquille eft épais, \& lorfqu'on le prend dans l'eau, avec l'Animal vivant, il cet de coulcur 


\section{DES COQUILLES. IZI}

couleur bleụe noirâtre foncée, quelquefois verdâtre; fon deffus eft raboteux : mais quand cette Coquille a été roulée dans le fable, telle qu'on la trouve dans les Jardins, elle a perdu une partie de fa couleur, \& $x$ il ne refte qu'un joli refeau, tantôt brun, tantôt rouge, quelquefois gris-de-lin, ou d'atitres nuances approchantes, fur un fond blanc.

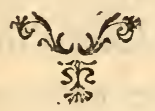




\section{L'Ancile. Ancylus.}

2 Tentacules.

Yeux placés à la bafe des tentacules, du côté intérieur.

Coquille univalve, Tefta univalvis, conconcave \& unie.

Tentacula 2.

Oculi ad bafim inter. ne.

cava, xqualis.

$\mathrm{I}$

ANCILE a un caractere fort approchant de celui du $\mathrm{Pla}-$ norbe.Il n'a, pareillement, que deux tentacules, \& fes yeux font placés à leur bafe, du côté intérieur. Mais ce qui diftingue ce genre de celui des Planorbes, \& de tous les autres, c'eft la forme de fa Coquille. Cette Coquille, faite comme un petit entonnoir plat \& allongé, ou comme une petite nacelle, n'a 
DES COQUILLES. I 23

aucunes fpirales; elle eft concave d'un côté, convexe en deffus, \& c'eft fous cette concavité qu'eft renfermé l'Animal, défendu par fa Coquille, quiil tient ordinairement appliquée contre les tiges des joncs. La pointe qui forme le fommet de la Coquille en deffus eft un peu recourbée de côté, \& elle n'occupe pas précifément le milieu de la Coquille. On trouve dans la Mer beaucoup de Coquilles de cette forme, connues fous le nom de Patelles, ou fous celui de Lepas. Mais comme leurs Animaux different un peu du nôtre par quelques caracteres,

F ij 
I24 T R A I T É

nous avons cru devoir donner à celui-ci un nom différent, \& nous l'avons appellé Ancylus, du mot Grec, A'zxúnos, qui fignifie convexe, à caufe de la forme de fa Coquille. Nous ne connoiffons ici qu'une feule efpece de'ce genre.

I. Ancylus.

Linn. Faun. Suec. 1293 . Patella, tefta membranea, ovali, mucrone reflexo.

Linn. Syf. Nat.edit. 1 o, $\dot{I}$, pag. 783 . n. 672 . Patella, tefta integerrima, ovali, membranea, vertice mucronato reflexo. Vulgò Lacuftris.

Lift. Angl. I I , to 2, $f_{0}$ 32. Patella 
DES COQUILLES. I 2$\}$ Aluviatilis, fufca, vertice mucronato inflexo.

Guale. Teft. t. 4, f. $A$ A.

Argenv. Conchyl. II, t. 8, f. $1, p . I$; t. $27, f . \mathrm{x}$.

L'Ancile: Longeur I $\frac{1}{2}$ ligne.

L'Ancile eft très petit comme on le voit par les dimenfions que nous en donnons. Sa Coquille eft mince, tranfparente \& très fragile. Sa pointe en deffus eft aiguë \& un peu recourbée. Ce petit Animal fe trouve dans les rivieres, attaché aux tiges de jonc; \& c'eft ainfi que l'a fait repréfenter $M$. d'Argenville, à la planche 27 , de la 
I 26 T R A I T premiere partie de fa Conchyliologie, f. I , quatrieme Lepas de cette figure.

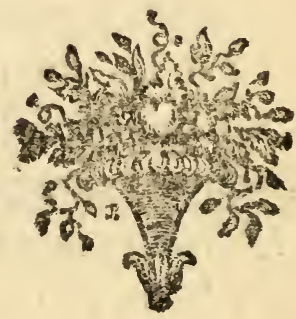




\section{SECTION SECONDE.}

COQUILLES BIVALVES.

Les Coquilles Bivalves font compofées de deux battants, affez femblables, entre lefquels eft renfermé l'Animal, \& qui s'ouvrent \& fe referment par le moyen d'une efpece de charniere. Comme ces Coquilles s'ouvrent peu, \& que l'Animal y eft adhérent \& n'en peut fortir, il n'eft pas auffi aifé de déterminer le caractere de ces Animaux, que celui de ceux des Coquilles univalves. Cependant

$$
\mathrm{F} \text { iv }
$$




\section{I28 T R A I T É}

on apperçoit quelques unes de lcurs parties, qu'ils font fortir hors de leurs Coquilles, lorfqu'on les examine dans l'eau. La plîpart ont des ouvertures, ou efpeces de fiphons, tantôt courts, tantôt pius allongés, quelquefois frangés, d'autres fois nuds, qu'ils font paroître, par le moyen defquels ils afpirent l'eau, \& avec clle différents corps qui leur fervent de nourriture, rejettant enfuice cette eau ou par le même fiphon nu par l'autre. Outre ces fiphons, on voit encore fortir de la Coquille, quelquefois à la partie oppofée, une autre partie fo- 


\section{DES COQUILIES. I 29}

lide, pluś ou moins allongée, qui paroît lui fervir de pied, \& qui en a reçu le nom de la plupart des Naturaliftes. Ce pied fert à la Coquille pour fe mouvoir \& changer un peu de place: je dis un peu; car en général ces Animaux ne font pas beaucoup dè chemin; il y cn a même qui reftent toute leur vie attachés au même rocher; telles font les huitres. C'eft d'après la forme des fiphons dont nous venons de parler, que nous avons tiré le caractere des Animaux qui habitent les Coquilles bivalves. Les Coquilles elles-mêmes nous ont fourni un autre caractere. 
I30 T R A I T É

Ces Coquilles, comme nous l'avons dit, font réunies par une efpece de charniere, qui varie pour la forme : tantôt elle eft unie \& attachée feulement par une membrane affez forte, tantô: celle eft garnie de dents en plus ou moins grande quantité, qui s'emboitent les unes dans les autres. Enfin un dernier caractere, fe prend dela forme même de la Coquille.

Les Animaux qui habitent ces Coquilles font hermaphrodites; ilsréuniffentles deux fexes:mais, bien différens des Limas \& des Buccins qui font pareillement hermaphrodites, on n'apperçoit 


\section{DES COQUILLES. I3I}

en les examinant, aucunes parties du fexe, foit mâles, foit femelles. Ils engendrent feuls fans accouplement marqué. Cette efpece de production étoit néceffaire pour desAnimaux dont plufieurs font immobiles \& conftamment attachés au même endroit. S'ils cuffent été diftingués de fexe, ou s'ils euffent eu befoin d'un double accouplement, comme le pratiquent les Limas, quoiqu'hermaphrodites, leur reproduction feroit devenue impraticable.

Parmi ces Animaux les uns font ovipares, les autres au contraire font vivipares, \& produi-

$$
\text { F vj }
$$


$132-T$ R A I T

fent des petits tout vivants, qui naiffent avec leurs petites Coquilles. Nous avons des exemples de ces deux efpeces de générations dans le peu de Coquilles bivalves qui fe trouvent aux environs de Paris. Ces Coquilles fe réduifent à deux feuls genres, la Came \&z la Moule, que nous allons examiner, \& qui font aquatiques, ainfi que toutes les bivalves. 
La CAME. CHA

2 Siphons, fimples \& Siphones 2, fimplices allongés. elongati.

Charniere de la Co- Cardo teft $x$ dentatus. quille dentelée.

Coquille arrondie. Tefta rotundata.

I. Chama, globofa glabra, cornei coloris, fulco tranfverfo.

Linn. Faun. Suec. n. 1336. Concha Nomen idem.

Linn. Syft. Nat. edit. 10, I, p. 678, n. 57. Tellina. Nomen idem.

Lift. Ang!. I so, tab. $2, f_{3}$ I. Mufculus exigutus, pifi magnitudine, rotundus, fubflavus, ipfis valvarum oris albidis.

Argenv. Conch. I, tab. 27, f. 9, n. 4 . Idom, Conchyl. II, t. $8, f .10$. 
?34 T R A I T É

La Came des Ruisseaux. Largeus $5,7,8$ lignes.

Cette petite Came varie beaucoup pour la grandeur, comme on le voit par les dimenfions que nous en avons données. Elle eft liffe en dehors, \& fa couleur eft pâle, un peu jaunâtre, prefque comme celle de la corne. Si on prend ce Coquillage vivant, \& qu'on le mette dans un bocal plein d'eau, il fair bientôt fortir d'un côté de fa Coquille un pied un peu allongé, \& de l'autre, deux fiphons dont les bords font unis, \& dont les cavités fe réuniffent enfemble. 


\section{DES COQUILIES. I 39}

C'eft par ces Siphons qu'on lui voit afpirer Zzrejetter l'eau, avec laquelle il attire quelques brins de mouffes, ou de petites plantes aquatiques qui lui fervent de nourriture. Mais une autre particularité, c'eft quefouvent dans ce même bocal, on le voit accoucher d'autres petits Coquillages vivants. Ainfi cette Came eft vivipare.Si on fépare les deux battants de la Coquille, on apperçoir à leur charniere deux petites dents. Les deux battans de la Coquille font égaux, élevés, renflés $\&$ arrondis. On trouve très communément cet Animal 


\section{T R A I T É \\ dans la riviere des Gobelins, \& dans les ruiffeaux des environs de Paris.}

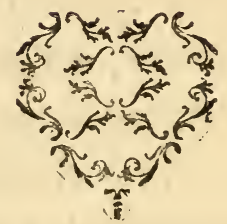




\section{La Moule. Mytulus.}

2 Siphons courts \& Siphones 2 , fimbriati frangés.

Charniere de la Co- Cardo teft membraquille membraneu- naceus, edentulus. fe \& fans dents.

Coquille allongée. Tefa elongata.

$\mathrm{N}$ voit, par les caracteres que nous donnons de la Moule, qu'elle differe de la Came par trois endroits effentiels. Le premier eft la forme de fes Siphons, qui font frangés à leur extrémité, \& fort courts; au lieu que ceux de la Came font longs \& fans aucune frange. Le fecond eft la ftructure de fa charniere qui n'a point de dents, mais une fimple rainure longue, dans la- 
I3s T R A I T É

quelle entre une efpece de feuillet mince; mais au lieu de ces dents, cette charniere eft affermie par une forte membrane, qui eft à l'extérieur de la Coquille. Enfin, la forme de la Coquille, qui eft allongée dans la Moule, eft le dernier caractere qui la diftingue de la Came, dont la Coquille eft courte \& arrondie. La Moule fe fert de fes fiphons de même que la Came; c'eft-à-dire qu'elle afpire l'eau par leur moyen, \& la rejette enfuite, après en avoir tiré fa nourriture. Cet animal eft ovipare; au lieu que la Came eft viviparc. Nous n'avons autour de 


\section{DES COQUIL LES. 139}

Paris, que les deux elpeces fuivantes.

I. Mytulus, tefta tenui, è fufco viridefcente, umbone non prominulo.

Linn. Faun. Suec, n. I332. Concha, tefta oblonga, ovata, longitudinaliter fubrugofa, poftice compreffoprominula.

Linn. Syft. Nat. edit. 1o, $I, p .706$, n. 21 \%. Mytulus, tefta ovali, compreffiufcula, fragiliffima, margine membranaceo, natibus decorticatis.

Lif. Angl. p. $146, t .2, f .29$. Mufculus latus, tefta admodum tenui, è fufco viridefcens, interdum rufefcens. 
$\$ 40$ T R A I T É

Argenville, Conch. $I, 2 a b .27, f .10$, n. $5,6,7$.

Idem, Conch. $I I, t .8, f_{0} 12$.

La Grande Moule des Etangs. Long. $6 \frac{1}{2}$ pouc. larg. $3 \frac{1}{2}$ pouc.

Cette grande Moule eft en dedans d'une très belle couleur nacrée, \& on apperçoit quelquefois dans fon intérieur quelques élévations, comme des perles. En dehors elle eft d'un brun verdâtre, \& lorfqu'on la regarde à travers le jour elle paroît tranfparente \& mince. L'endroit de fa charniere n'eft nullement prominent, \& fe trouve plus près d'un des côtés, à peu 


\section{DES COQUILLE. I4}

près à un tiers du bord de la $\mathrm{Co}-$ quille. Le deffus de cette Coquille a beaucoup de fillons, grands, tranfverfes \& concentriques à l'endroit de la charniere. On trouve cette Coquille dans les étangs. C'eft fans contredit la plus grande de toutes celles de ce Pays-ci.

II. Mytulus, tefta fufca, umbone prominente.

Lift. Ang. 149, t. 2, f. 30. Mufculus anguftior, ex flavo viridefcens, validus, umbonibus acutis, valvarum cardinibus, velut pinnis donatis finuofis.

Argenv. Conch. I, t. $27, f .10, \pi .4$. 
142 T R A I T E

Idem, Conchyl. II, t. 8, f. II.

La Moule des Rivieres. Longueur I $\frac{1}{2}$ pouce, largeur 10 lignes.

Cette Moule reffemble beaucoup à la précédènte, à la grandeur près; cependant on y trouve plufieurs différences. Premierement la couleur de la Coquille en dehors eft plus brune, tirant fur le verd brun, \& quelquefois fur le noir. Secondement, l'endroit de la charniere eft plus éminent \& beaucoup plus aigu que dans la grande Moule. Enfin, le deffous de la charnicre, à l'intéricur fous cettéminence, forme un enfon- 


\section{DES COQUILLS. 43}

cement confidérable, accompagné, à côté, d'une autre cavité moins grande. On trouve cette Coquille très communément dans les rivieres.

\section{F I N.}




(3)

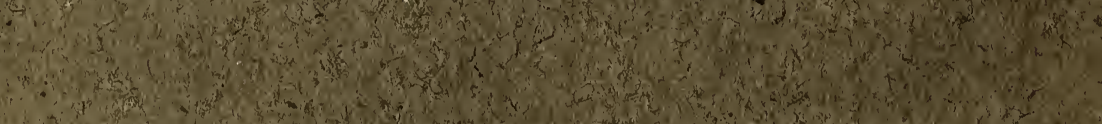

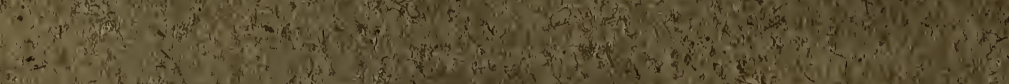

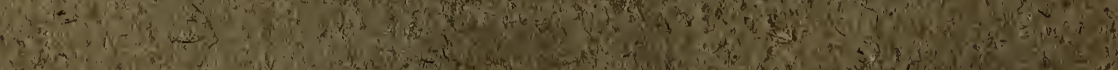

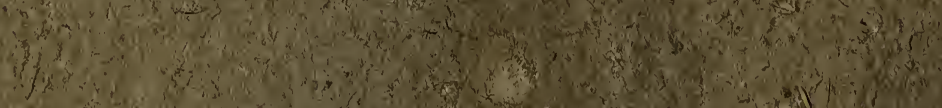

Xin

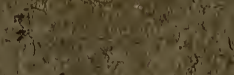

